\title{
GaAs Periodic Half Octagonal Cut Based Nano Texturized Hexagonal Shaped Nanopillar Array Structure for Highly Responsivephotodetector's Performance
}

\section{Smriti Baruah ( $\square$ sbbaruah1993@gmail.com )}

North Eastern Regional Institute of Science and Technology https://orcid.org/0000-0003-0153-1084

\section{Santanu Maity}

IIEST Shibpur: Indian Institute of Engineering Science and Technology

Joyatri Bora

NERIST: North Eastern Regional Institute of Science and Technology

\section{Research Article}

Keywords: Photodetector, nanopillar, quantum efficiency, half octagonal, absorption, responsivity

Posted Date: March 11th, 2021

DOl: https://doi.org/10.21203/rs.3.rs-273099/v1

License: (c) (1) This work is licensed under a Creative Commons Attribution 4.0 International License.

Read Full License 


\title{
GaAs periodic half octagonal cut based nano texturized hexagonal shaped nanopillar array structure for highly responsivephotodetector's performance
}

\author{
Smriti Baruah*, Santanu Maity ${ }^{1}$ and Joyatri Bora ${ }^{2}$ \\ ${ }^{*}, 2$ Department of Electronics and Communication Engineering, North Eastern Regional Institute of \\ Science and Technology, India \\ ${ }^{1}$ Centre of Excellence for Green Energy and Sensor Systems, Indian Institute of Engineering Science \\ and Technology, Shibpur, Howrah-711103, India \\ *sbbaruah1993@gmail.com, ${ }^{1}$ maitys.iiest@gmail.com, ${ }^{2}$ jb@nerist.ac.in,
}

\begin{abstract}
:
Efficient light absorption is the most crucial factor for high spectral responsive photodetection phenomenon. Nanopillars for photodetection constitute a great opportunity in enhancing the light absorption efficiency along with reducing the electrical cross-section of the device. Here an analytical investigation of a proposed periodic GaAs material based half octagonal cut based vertically oriented hexagonal nanopillar array deployed over a 5 um x 5 um front detector's surface has been done for attaining an optimum light absorbance by reducing the surface reflectivity of the incoming photons. The geometrical analysis of the proposed model exhibits a 0.999 absorbance and $0.97 \mathrm{~A} / \mathrm{W}$ photoresponsivity along with $86 \% \mathrm{EQE}$ at 1 um operating wavelength.
\end{abstract}

Keywords: Photodetector; nanopillar; quantum efficiency; half octagonal; absorption; responsivity

\section{Introduction}

Currently there is a rapid growing advancement in a large number of technological platforms for which photodetectors are very crucial element [1,2]. Some of the established platforms include solar cell, fibre optical communications as well as the profound emerging technologies such as laser radar (LIDAR) for self-driving cars, free space optical communications, and advanced image sensors [3]. In order to attain an optimum performance efficiency of these devices the absorption spectrum of the photodetector should be its key stipulation. It is emanated from a combination of efficient light absorption spectrum and quantum efficiencies [4]. For a planar bulk unstructured detector's planar surface the absorption efficiency is at maximum $70 \%$ due to the complex higher refractive index mismatch between the air semiconductor interface $[5,6]$. Therefore, in order to boost up this absorption efficiency several approaches have been adopted in terms of III-V nanostructures as they arouse as a promising candidate in minimizing this lattice mismatch owing to their small that could make the lattice mismatch to relax horizontally near the base [7, 8]. Among these nanostructures, vertical nanopillar structure has emerged as the most significant result in silicon compatibility which could easily resolve the issues of CMOS BEOL incompatible temperature associated with quantum dots and high nonradiative recombination at the surface in nanowire [9]. Nanopillars could be grown without catalysts thus eliminating the metal contamination effect [10]. Moreover, they could grow in a core-shell deposition like manner which could enable them to grow in micron sizes without introducing any defects [11]. Numerous researches are going on single and array based nanopillars structures for obtaining high quantum efficiency, lower dark current and to attain maximum photoresponsivity in photodetection phenomenon. Several fabrication methods have been adopted where the values exhibits a broader band suppression of reflectance that lead towards towards spectacular luminous intensity magnitude for more than 1 [12]. Along with the wider spectrum of optical radiation that has been covered for various applications, vertical nanopillars played a key role in infrared sensing applications leading towards a high quantum efficiency of up to $29 \%$ with the high operating system at $2300 \mathrm{~nm}$ that is well beyond the diffraction limit [13]. The vertical orientation of nanopillar provides a large surface to radial junction along with increasing path length of incident light. Also the reduced reflectivities resulting towards higher absorbing volume for maximum carrier absorption and collection in comparison to their single counterpart $[14,15]$. In this respect several approaches have been done with III-V based nanopillar 
to boost the photodetector performance parameters by minimizing the surface reflectivity. Some of them include InP based nanopillar arrays that have been fabricated from epitaxially grown InP/GaInAsP/InP quantum well provides outstanding photoluminescence [16]. GaP nanoarrays surface prepared by the chemical vapor deposition method also depicts reduced reflectivity for a broader angular and wavelength range [17]. Singlelayer GaAs nano pyramids that have been prepared by a combination of lithography, Metal enhanced chemical vapor deposition method and gas-phase substrate also showed remarkable absorption at wider radiation spectrum and the broad incident angle at wider curvature bending [18].

Although an ample amount of research have been done over the photodetector's suface light reflectance mitigation using several nanopillar based techniques however, periodic nano texturization over the nanopillar structures engulfing the concept of the internal multiple reflection phenomenon for reducing the overall light reflectance has not been discussed earlier in length.

In the prior work, authors have demonstrated the effect of GaAs material based nanotextured pyramidal cut nanopillar array where the multiple internal reflection phenomenon for the reflectance mitigation takes place within the interpillar spacing of the four adjacent nanopillar faces [19]. In [20] InGaAs based hexagonal shaped nanopillar has been adopted for light reflectance minimization that provides the internal multiple reflection phenomenon to take place within six nanopillar interfaces instead of four. This enhances the total amount of overall photon absorption In this paper the authors have proposed a model consisting of periodic half octagonal cut based texturization over the hexagonal shaped nanopillar array so as to reduce the light reflectance phenomenon to a minimal level. The half octaganol cut nanotexturization over the six faces of the hexagonal nanopillar would further enhance the amount of multiple internal reflections for photon reabsorption within the photodetector device in comparison to [20]. Also the six adjacent faces of the proposed nanopillar structure would grasp a higher quantity of photons within them in comparison to other models. The structural parameters of the proposed model including the bending angles of the half octagonal cut, optimal interpillar spacing and the nanopillar striking angles have been analytically investigated and modelled so as to trap the incoming light as much as possible within the proposed nanopillar array. Section II demonstrates the optical modelling of the proposed structure. The photovoltaic characterization as well as the effect of the proposed nanopillar structural parameters over the photodetector's performance metrics has been demonstrated in the form of simulative representations in section III.

\section{Optical modelling of the NP array structure:}

Figure 1(a) provides the 3D schematic of the proposed periodic half octagonal cut nano textured GaAs based hexagonal nanopillar array with ' $h$ ' pillar height, planar detector's surface width ' $w$ ' and 's' being the interpillar gap deployed over a 50um $x$ 50um detector's front surface area. To demonstrate the light reflectance behavior of the incoming photons inside the nanopillar array geometric ray analysis has been adopted. For simplicity incoming photons at an operating wavelength of $850 \mathrm{um}$ has been considered to get emitted from a $2 \mathrm{~mm}$ diameter GaAs LED source. The transformation of half octagonal nanotextured cut from previously proposed pyramidal cut by increasing the centre bending angle $\left(\theta_{\mathrm{B}}\right)$ has been depicted in Figure 1(b). 


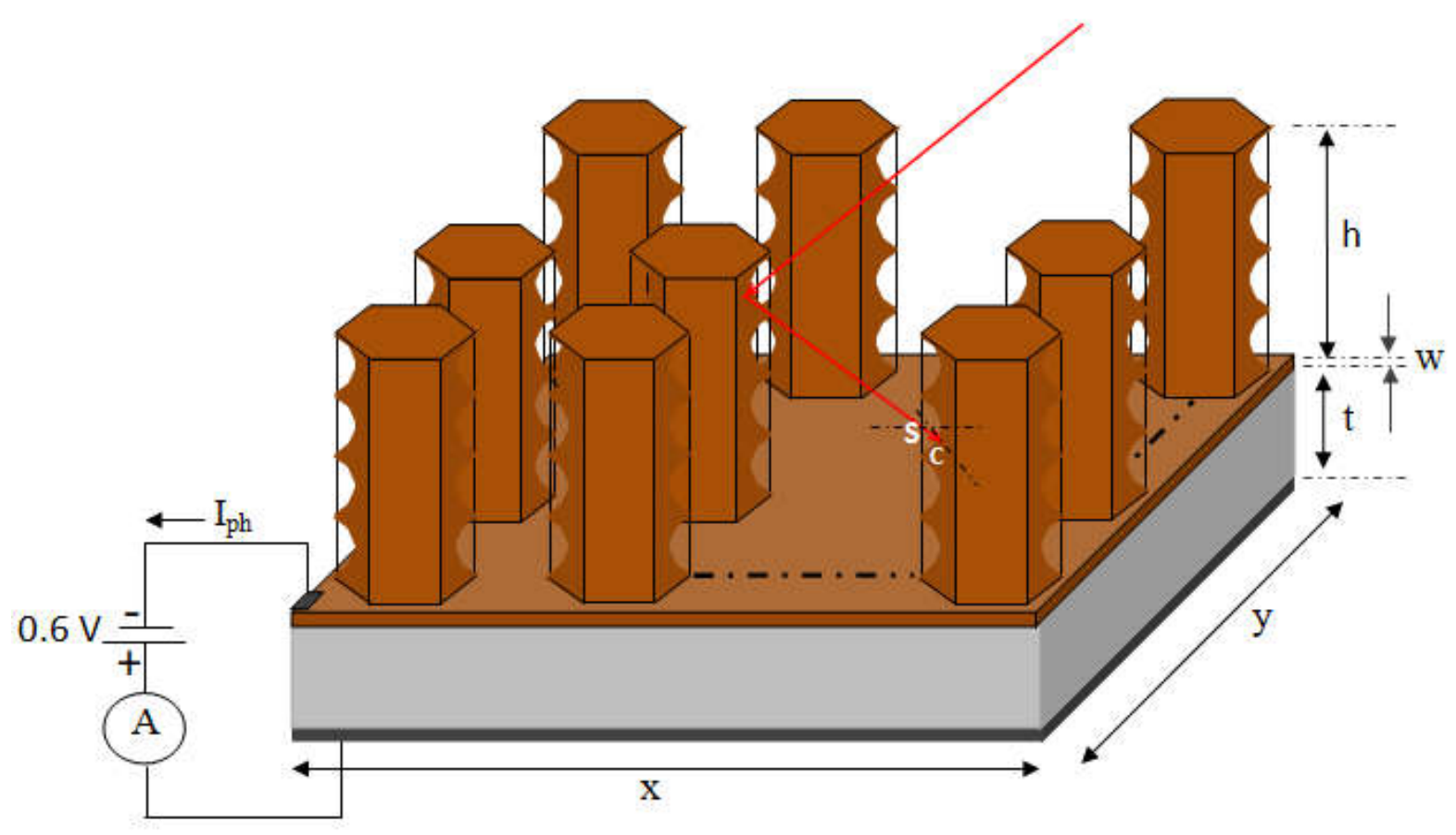

(a)

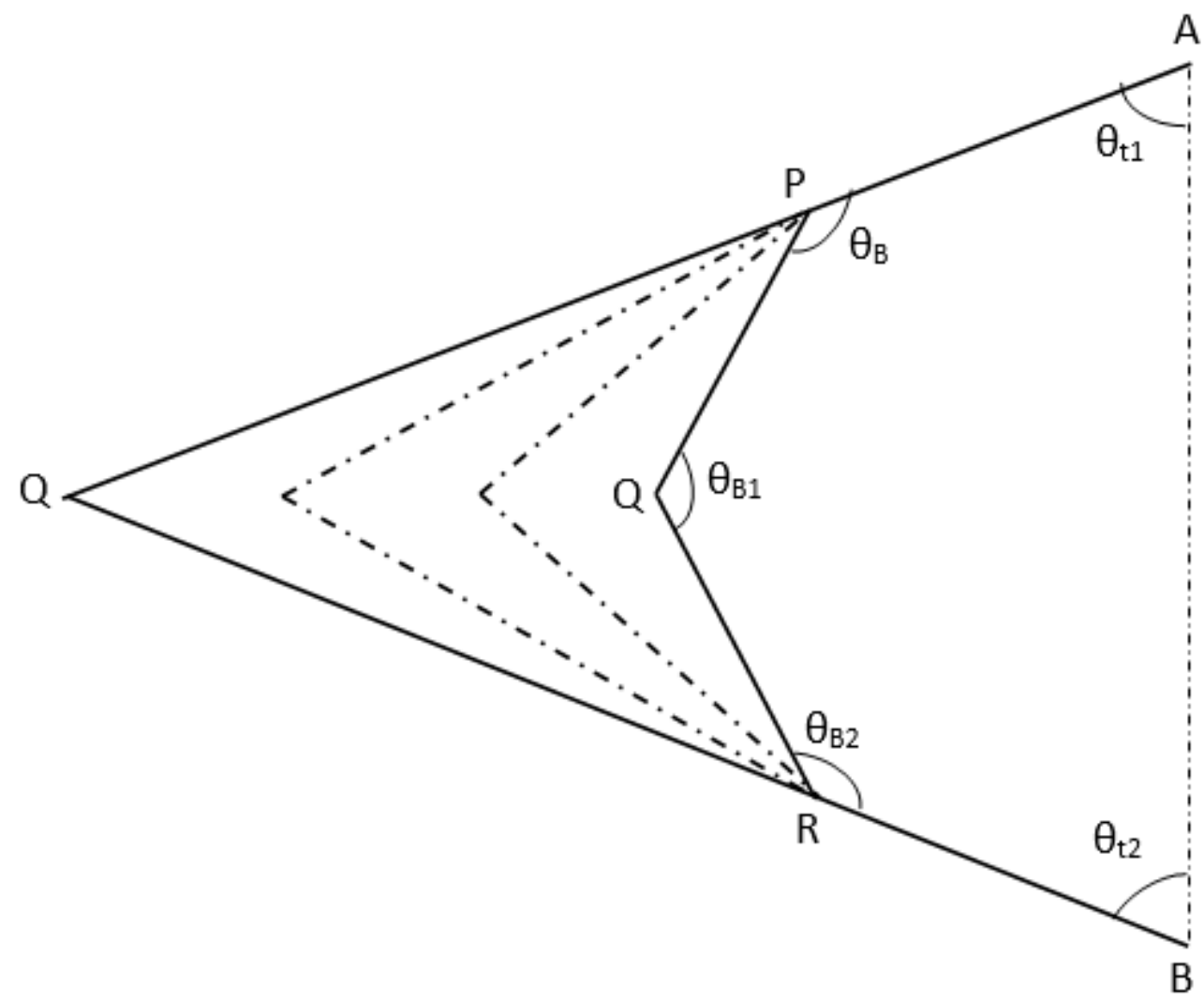

(b)

Fig. 1 (a) Light interaction pattern within a proposed GaAs based half octagonal cut based nanotextured hexagonal-shaped nanopillar array photodetector surface structure, and (b) Schematic showing the half octagonal cut transformation from a pyramidal shaped cut. 
For the easier understanding of the ray analysis pattern, Fig. 2 demonstrate the striking angles formation of the incoming photons at the proposed half octagonal cut based nanopillar interface w.r.t their incident angle at the planar detector's surface. The half octagonal textured cut at the hexagonal nanopillar depends on the three bending angles $\left(\theta_{\mathrm{B}}, \theta_{\mathrm{B} 1}, \theta_{\mathrm{B} 2}\right)$ as shown in the Figure.

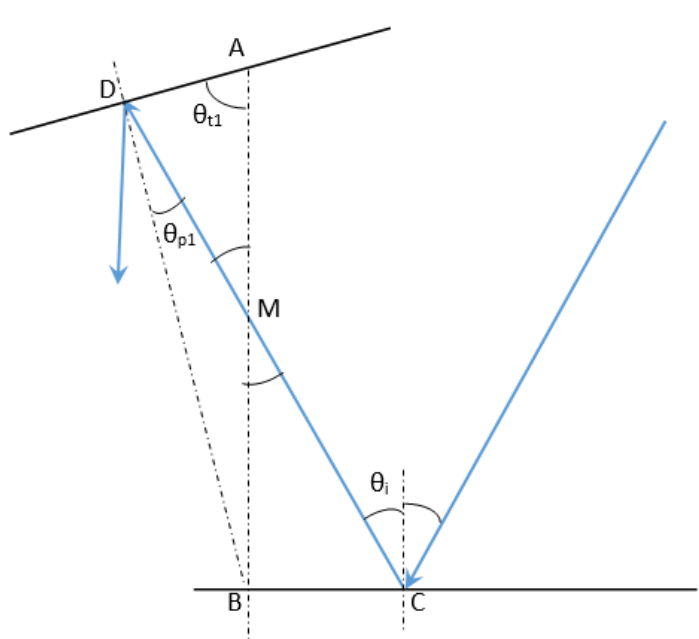

(a)

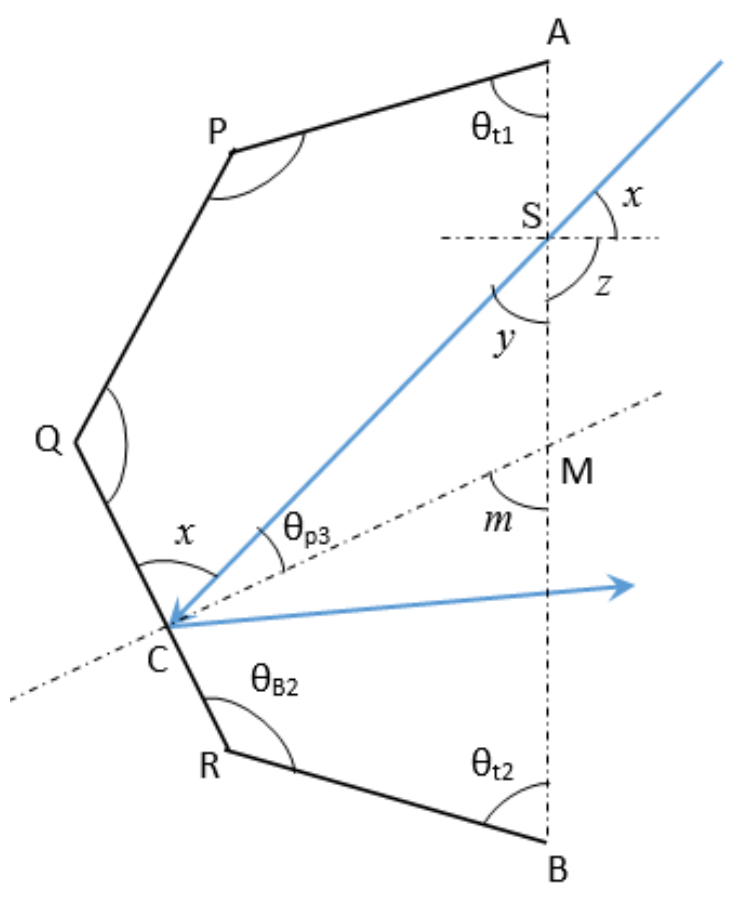

(c)

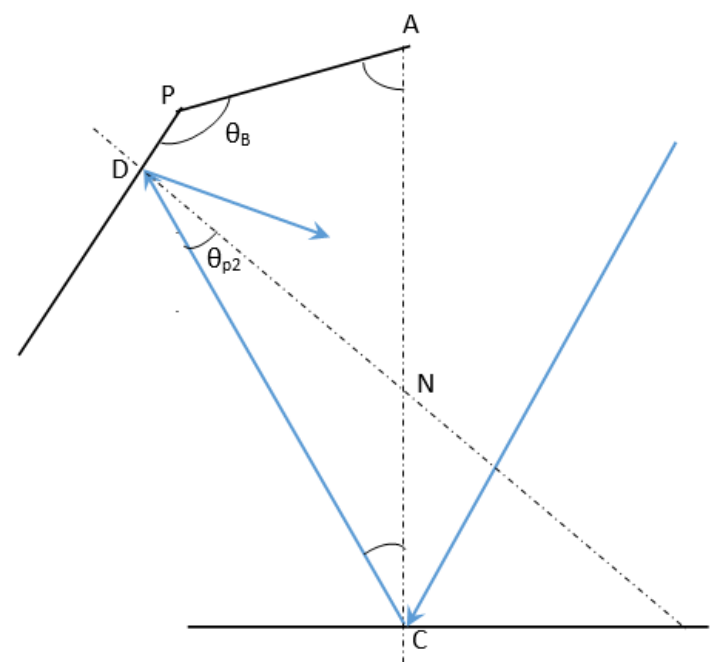

(b)

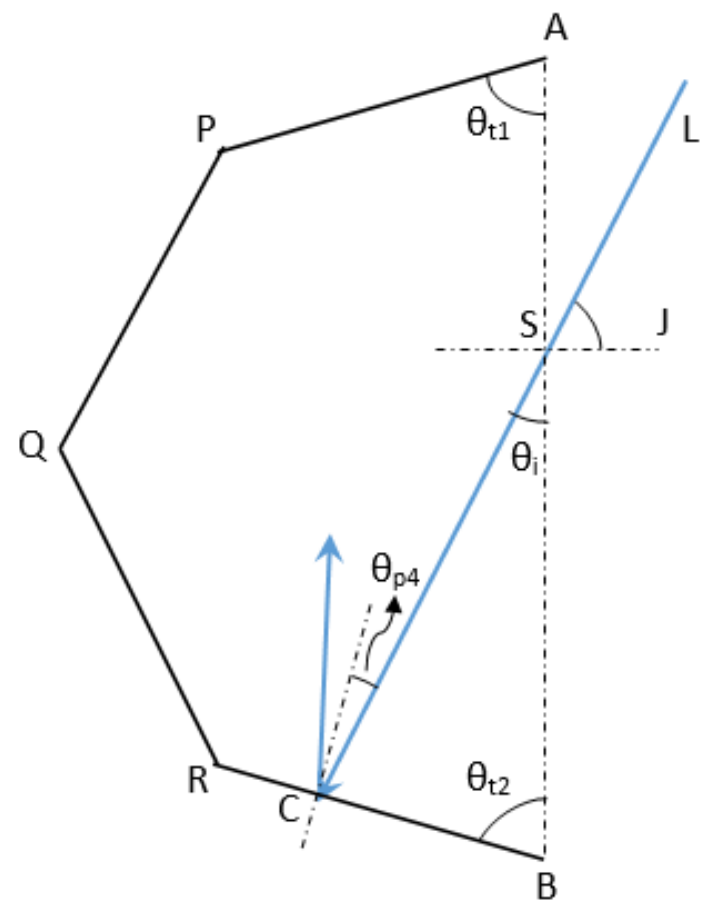

(d)

Fig. 2. Schematic representing the light striking angle formation: (a) $\theta_{\mathrm{p} 1}$ at first upper interface, (b) $\theta_{\mathrm{p} 2}$ at second upper interface, (c) $\theta_{\mathrm{p} 3}$ at first lower interface, and (d) $\theta_{\mathrm{p} 4}$ at second lower interface w.r.t incoming light incident angle $\left(\theta_{\mathrm{i}}\right)$

As could be demonstrated from the Fig. 2(a) the incoming photon that incident at the planar detector's surface with ' $\theta_{\mathrm{i}}$ ' incident angle after reflection hits the upper $\left(\theta_{\mathrm{t} 1}\right)$ tilted surface of the proposed pentagonal shaped nanopillar cut with an angle $\left(\theta_{\mathrm{p} 1}\right)$ at point ' $\mathrm{D}$ '. The value of this striking angle could be deduced with Fig. 2(a) as, 
In $\triangle \mathrm{BMC}$,

$$
\begin{aligned}
& \angle B M C+\angle M B C+\angle M C B=180^{\circ} \\
& \Rightarrow \angle B M C+\angle 90^{\circ}+\angle 90^{\circ}-\angle \theta_{i}=180^{\circ} \\
& \Rightarrow \angle B M C=\angle \theta_{i}=\angle D M A
\end{aligned}
$$

Now, In $\triangle$ MDA,

$$
\begin{aligned}
& \angle D M A+\angle M D A+\angle D A M=180^{\circ} \\
& \Rightarrow \angle \theta_{i}+\angle M D A+\angle \theta_{t 1}=180^{\circ} \\
& \Rightarrow \angle M D A=180^{\circ}-\left(\angle \theta_{i}+\angle \theta_{t 1}\right)
\end{aligned}
$$

Now,

$$
\begin{aligned}
& \angle 90^{\circ}-\angle M D A=\angle \theta_{p 1} \\
& \Rightarrow \angle 90^{\circ}-\left(180^{\circ}-\left(\angle \theta_{i}+\angle \theta_{t 1}\right)\right)=\angle \theta_{p 1} \\
& \Rightarrow \angle \theta_{p 1}=\angle \theta_{i}+\angle \theta_{t 1}-90^{\circ}
\end{aligned}
$$

Figure 2(b) demonstrates the formation of the striking angle $\left(\theta_{\mathrm{p} 2}\right)$ of the reflected portion of the incoming photon ray at the second upper interface of the proposed nanopillar structure at point ' $\mathrm{D}$ '. The value of this striking angle could be deduced as follows,

In quadrilateral APDC (Fig. 2(b)),

$$
\begin{aligned}
& \angle C A P+\angle A P D+\angle P D N+\angle N D C+\angle D C A=360^{\circ} \\
& \Rightarrow \angle \theta_{t 1}+\angle \theta_{B}+90^{\circ}+\angle \theta_{p 2}+\angle \theta_{i}=360^{\circ} \\
& \Rightarrow \angle \theta_{t 1}+\angle \theta_{B}+\angle \theta_{p 2}+\angle \theta_{i}=180^{\circ} \\
& \Rightarrow \angle \theta_{p 2}=180^{\circ}-\left(\angle \theta_{t 1}+\angle \theta_{B}+\angle \theta_{i}\right)
\end{aligned}
$$

For the incoming photon striking the first lower proposed nanopillar interface directly at point ' $\mathrm{C}$ ' without hitting the planar detector's surface is illustrated in Fig. 2(c). The incoming photons forms an angle ' $x$ ' at point ' $S$ '. This angle represents the formation of the striking angle of the incoming photon initially at the vertical nanopillar interface without any octagonal cut.The value of this striking angle could be calculated as [19],

$$
\angle x=90^{\circ}-\theta_{i}
$$

The striking angle $\left(\theta_{\mathrm{p} 3}\right)$ formed w.r.t to the octagonal cut would be of the form, 
In quadrilateral BRCM (Fig. 2(c)),

$$
\begin{aligned}
& \angle \theta_{t 2}+\angle \theta_{B 2}+\angle 90^{\circ}+\angle m=360^{\circ} \\
& \Rightarrow \angle m=180^{\circ}-\left(\angle \theta_{t 2}+\angle \theta_{B 2}\right)
\end{aligned}
$$

Now, $\angle x+\angle y+\angle z=180^{\circ}$ (Angles of a line)

$\Rightarrow\left(90^{\circ}-\angle \theta_{i}\right)+90^{\circ}+\angle y=180^{\circ}$

$\Rightarrow \angle y=\angle \theta_{i}$

In $\triangle C M S$,

$\angle \theta_{p 3}+\angle y+180^{\circ}-\angle m=180^{\circ}$

$\Rightarrow \angle \theta_{p 3}+\angle \theta_{i}+180^{\circ}-180^{\circ}+\angle \theta_{t 2}+\angle \theta_{B 2}=180^{\circ}$

$\Rightarrow \angle \theta_{p 3}+\angle \theta_{i}+\angle \theta_{t 2}+\angle \theta_{B 2}=180^{\circ}$

$\Rightarrow \angle \theta_{p 3}=180^{\circ}-\left(\angle \theta_{i}+\angle \theta_{t 2}+\angle \theta_{B 2}\right)$

The angle formed by the incoming ray striking the second lower textured nanopillar cut at point ' $\mathrm{C}$ ' could be deduced with Fig. 2(d) as:

In $\triangle \mathrm{BCS}$,

$\angle B S C+\angle B C S+\angle C=180^{\circ}$

Also,

$$
\begin{aligned}
& \angle B S C=\angle L S A=\theta_{i} \\
& \Rightarrow 90^{\circ}-\angle \theta_{p 4}+\theta_{i}+\angle \theta_{t 2}=180^{\circ} \\
& \Rightarrow \angle \theta_{p 4}=\angle \theta_{i}+\angle \theta_{t 2}-90^{\circ}
\end{aligned}
$$

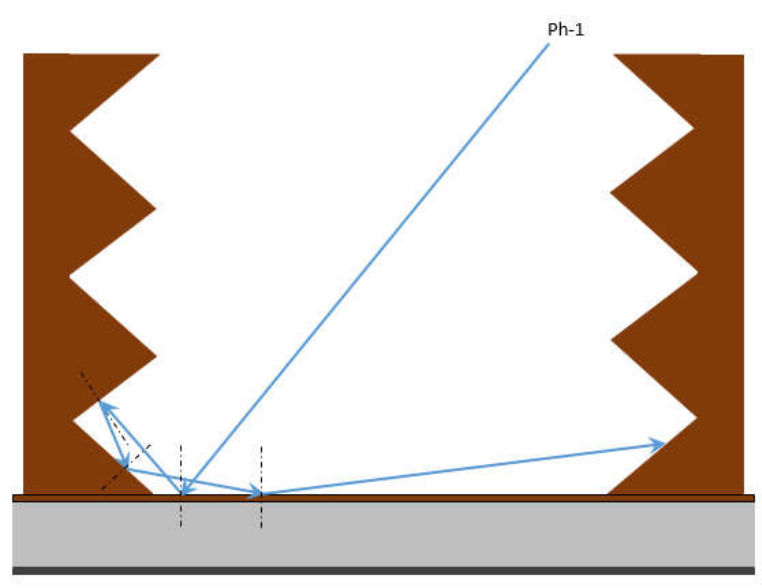

(a)

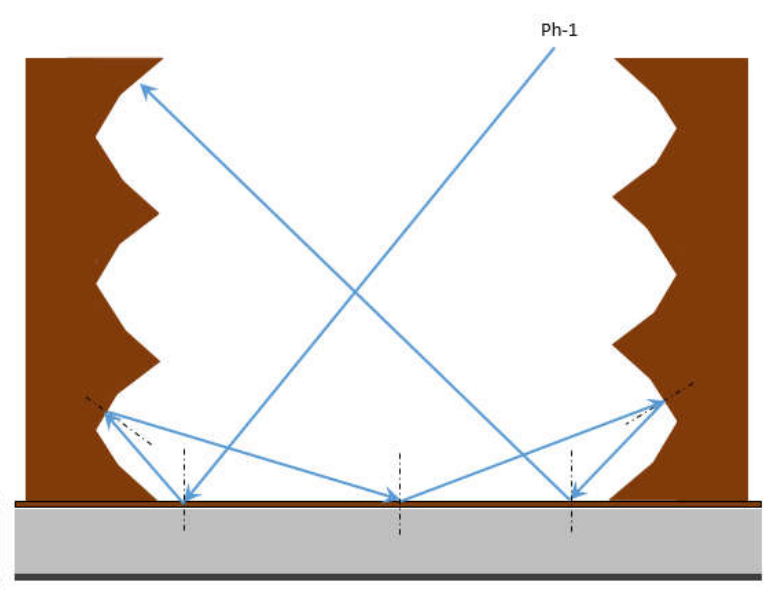

(b) 


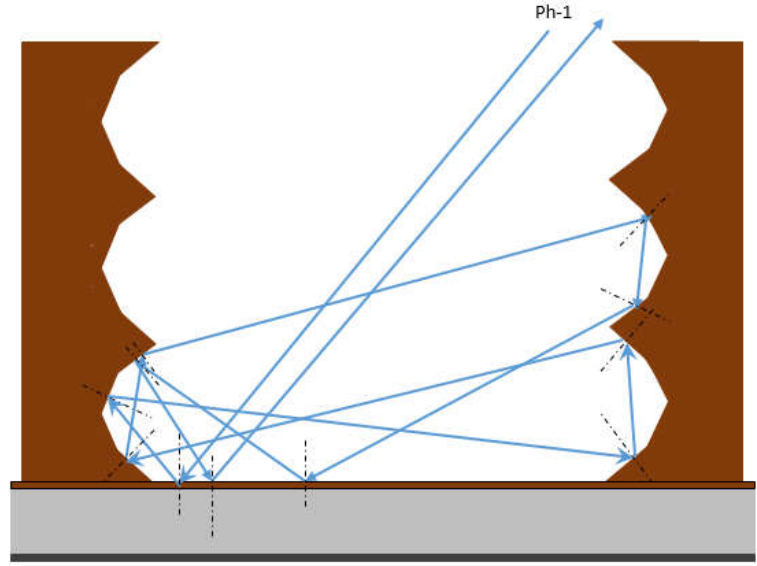

(c)

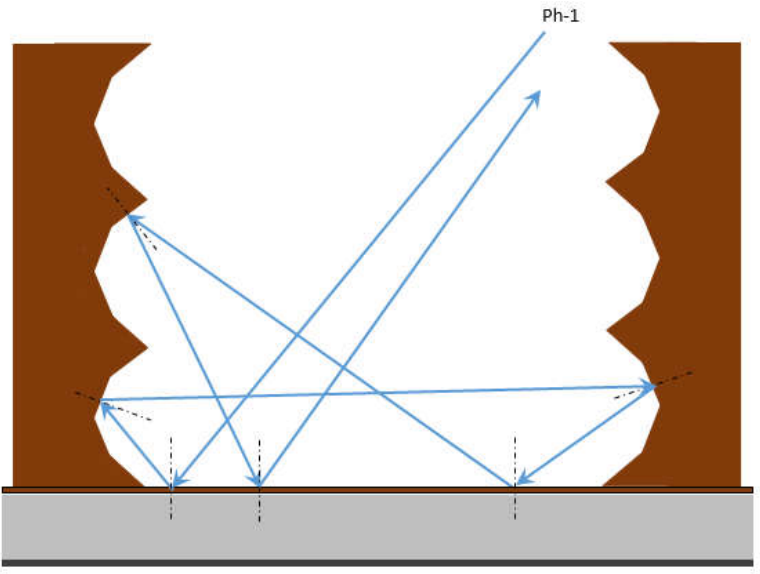

(d)

Fig. 3. Light reflectance pattern within the proposed octagonal cut based nanopillar array structure with centre bending angle $\left(\theta_{\mathrm{B}}\right)$ of: (a) $45^{\circ}$, (b) $100^{\circ}$, (c) $140^{\circ}$, and (d) $160^{\circ}$

Figure 3 compares the light reflectance followed within the proposed pentagonal nanopillar array with four different structural models (Model-I, Model-II, Model-III and Model-IV) consisting of varying bending angles $\left(\theta_{\mathrm{B}}, \theta_{\mathrm{B} 1}, \theta_{\mathrm{B} 2}\right)$. Figure 3(a) demonstrates the light reflectance pattern within the Model-I pyramidal shaped nanopillar array structure with a nanopillar bending angle $\left(\theta_{\mathrm{B}}\right)$ of $45^{\circ}$ along with the other two bending angles $\left(\theta_{\mathrm{B} 1}\right.$ and $\left.\theta_{\mathrm{B} 2}\right)$ being $180^{\circ}$. As the figure shows, the single incoming photon ph-1 strikes the flat detector's front surface with an incident angle $\left(\theta_{\mathrm{i}}=40^{\circ}\right)$. The reflected portion of the incoming ray from point 'a' undergoes five internal reflections within the inter pillar gap at various points. This multiple reflection phenomenon would enhance the reabsorption probability of the incoming photon within the device which would otherwise have been lost after a single reflection from the planar photodetector's front surface.

Figure 3(b) represents the pentagonal cut textured nanopillar structural Model-II with ' $\theta_{\mathrm{B}}$ ' of $100^{\circ}$ and other bending angles ' $\theta_{\mathrm{B} 1}$ ' and ' $\theta_{\mathrm{B} 2}$ ' of $150^{\circ}$ each. As could be observed from the figure with increasing centre bending angle ' $\theta_{\mathrm{B}}$ ' and decreasing ' $\theta_{\mathrm{B} 1}$ ' and ' $\theta_{\mathrm{B} 2}$ ' tilted angles the nanopillar cut took the shape of a half octagon. The half octagonal cut assist in augmentation of the number of multiple reflections of the incoming photon ray taking place within the nanopillar array. In comparison to the pyramidal cut shaped nanopillar cut which could provide only two internal reflections of the incoming photon the half octagonal cut of the nanopillar could provide six internal reflections. With this increasing multiple reflection phenomenon the reabsorption probability of the incoming photon boost up to a significant level. This enhanced reabsorption minimizes the signal loss to lowest level.

Figure 3(c) provides the schematic of the third Model-III broader angled half octagonal cut based hexagonal nanopillar array structure. The textured half octagonal cut consist of a centre bending angle ' $\theta_{\mathrm{B}}$ ' of $140^{\circ}$.The incoming photon ray (ph-1) after reflecting from the front detector's surface undergoes maximum number of eleveninternal reflections within the inter pillar gap at various nanopillar striking points.This increased internal reflections enhanced the light trapping efficiency and ultimately the reabsorption probability to a maximal level.

However, increasing the bending angles beyond a certain limit would minimize the internal reflection phenomenon as depicted in Fig. 3(d). The half octagonal cut of this structural model consist of bending angles ' $\theta_{\mathrm{B}}$ ' of ' $160^{\circ}$ ' while ' $\theta_{\mathrm{B} 1}$ ' and ' $\theta_{\mathrm{B} 2}$ ' of $130^{\circ}$ each. As could be demonstrated from the figure, the secondary reflected ray after going six internal reflections within the proposed structure gets lost to air without much aiding towards total enhanced light absorbance. Thus, it has been perceived that in order to achieve maximum trapping efficiency of the incoming photons Model-III half octagonal cut texturization is the most suitable nanopillar structure.

The maximum number of half octagonal cut textured nanopillars that could be deployed over a '5um $x$ 5 um' photodetector's front surface area is [20],

$$
N_{p}\left(x_{n_{\text {tier }}} \times y_{n_{\text {tier }}}\right)=1+6 n_{\text {tier }}\left(n_{\text {tier }}+1\right) / 2+4\left(n_{\text {tier }}-1\right)
$$

Where, ' 1 ' represents the mother hexagonal pillar and ' $n_{\text {tier }}$ ' represents the total number of tiers within the nanopillar array with a value [20]. 


$$
n_{\text {tier }}=\frac{y-D}{2(D+d)}
$$

Here, ' $D$ ' denotes the hexagonal nanopillar diameter.

In order to obtain maximum light trapping efficiency inside the nanopillar structure there should be an optimum spacing between two adjacent nanopillars so as to allow the maximum incoming photons to get trap inside besides their maximal multiple internal reflections for enhanced detector's absorption. Figure 4 depicts four cross sectional view schematics of the broad bending angled half octagonal cut based textured hexagonal nanopillar array consisting of height ' $h$ ', upper and lower tilted cutting angle $\left({ }^{\prime} \theta_{t 1}{ }^{\prime}={ }^{\prime} \theta_{t 2}{ }^{\prime}=20^{\circ}\right)$, centre bending angle $\left(\theta_{B}=160^{\circ}\right)$ and varying interpillar gap ' $d$ ' to investigate the optimum interpillar spacing required based on the nanopillar height for maximum light absorption.

Figure 4(a) demonstrates the proposed nanopillar schematic representation consisting of an interpillar gap much smaller than that of the nanopillar height $(d<h)$.The analytical investigation of the optimum spacing has been done in terms of five incoming photons trapping inside the nanopillar array with $40^{\circ}$ planar surface incident angle. As could be evaluated from the figure, only two out of the seven incoming photons (ph-1, ph-2) got trapped inside the array. The first incoming photon hits the second lower nanopillar cut interface with an angle of $20^{\circ}$ in accordance to the Eqn. (7). After reflection from this point the reflected ray undergoes through another three internal reflections within the nanopillar array at various points as shown before getting finally lost to air. The reflected ray of the second incoming photon after reflecting from the detector's front planar surface undergoes a maximum of twelve internal reflections within the array before getting out. This interpillar gap would although provides a more amount of internal reflections to the first two incoming photons for enhanced absorption however only two of the five incoming photons could get trapped inside the array. The remaining three photons would require another pair of nanopillars which could decrease the cost efficiency.

Figure 4(b) depicts the cross section schematic of the pair of proposed nanopillar with an interpillar gap of $(h=d)$. Here three of the five incoming photons could be trapped inside the nanopillar array. With this nanopillar gap the first incoming photon undergoes four internal reflections, second one faces twelve while the third incoming undergoes five internal reflections within the array. Although a more incoming photon could be trapped still remaining two would be lost without aiding towards high detector's responsivity.

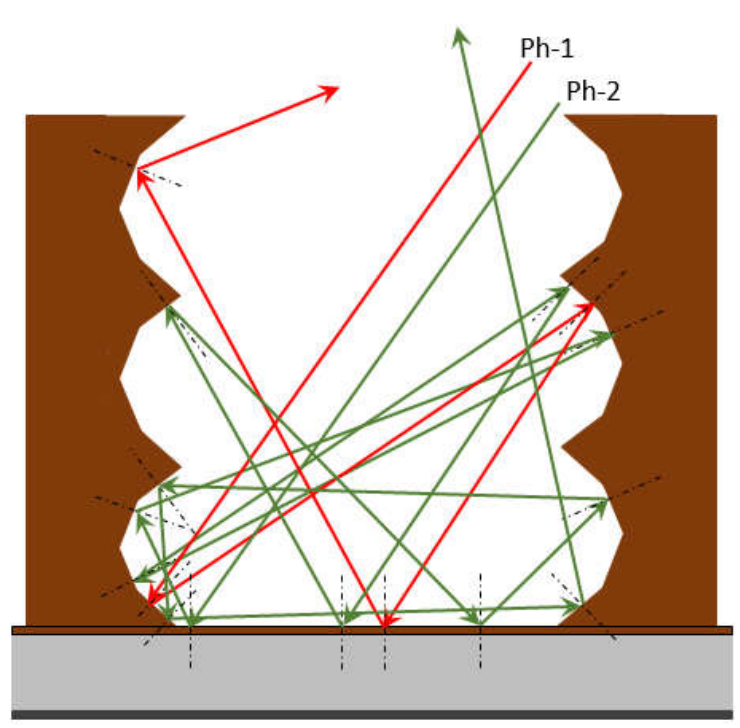

(a)

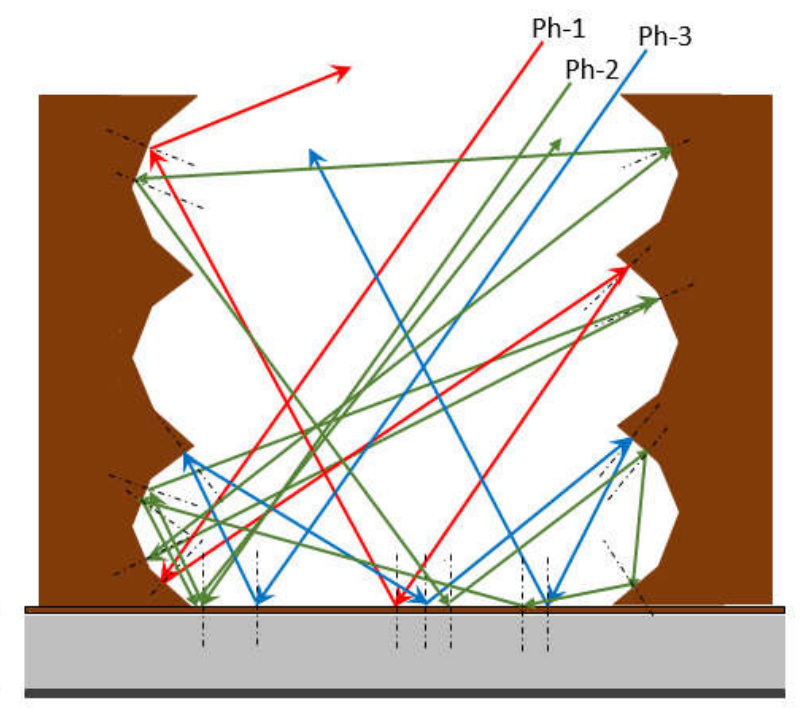

(b) 


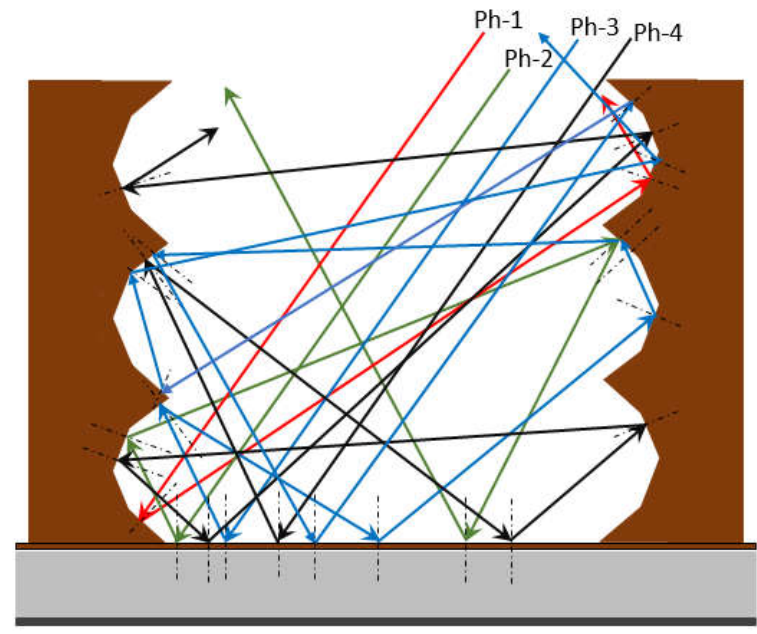

(c)

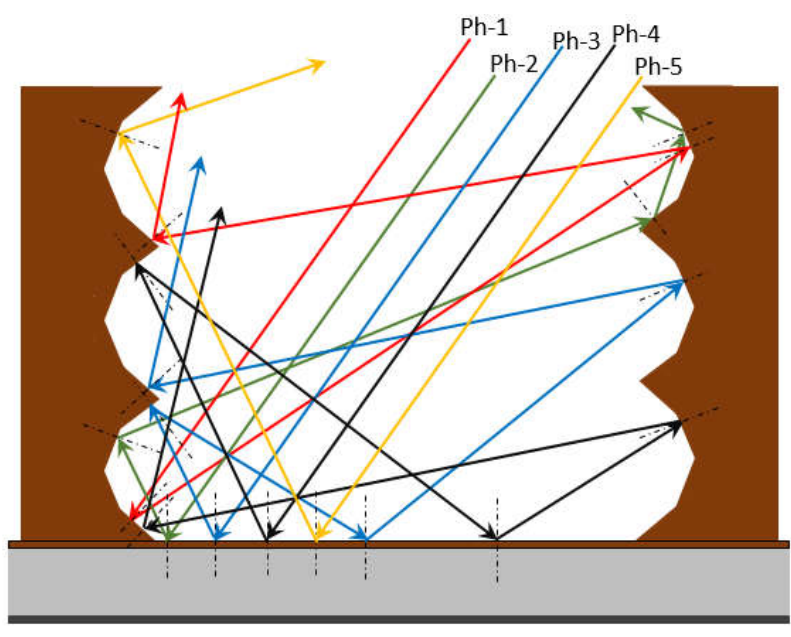

(d)

Fig. 4. Light reflectance pattern within the proposed octagonal cut based nanopillar array structure with varying interpillar spacing: (a) $d<h$, (b) $h=d$, (c) $d=\left[2\left(h-l \cos \left(\theta_{t l}\right)\right) / \tan \left(90^{0}-\theta_{i}\right)\right]-l \sin \left(\theta_{t I}\right)$, and $(d) d>[2(h-$ $\left.\left.\left.l \cos \left(\theta_{t l}\right)\right)\right) / \tan \left(90^{\circ}-\theta_{i}\right)\right]-l \sin \left(\theta_{t l}\right)$

The nanopillar pair schematic representation of Fig. 4(c) provides an interpillar spacing of a value,

$$
d=\frac{2\left(h-l \cos \left(\theta_{t 1}\right)\right)}{\tan \left(90^{\circ}-\theta_{i}\right)}-l \sin \left(\theta_{t 1}\right)
$$

Here, ' $l$ ' denotes the length of each of the side of the textured half octagonal cut.

This optimum interpillar gap allows the as much as incoming photons to get trapped within the nanopillar array such that the last incoming photon after reflection from the planar detector's surface could hit at the tip of the second nanopillar cut interface as shown in the figure. Out of the five considered incoming photons, four of the incoming photons have been depicted to get trapped within the array undergoing three, four, eleven, and eight internal reflections respectively. Although the first, second and third incoming photons undergoes through a bit lesser number of internal reflections with this interpillar gap in comparison to the previous ones however, maximum number of incoming photons could be trapped within and also the small reduction in reflection numbers could be neglected in comparison to the maximum allowable trapped incoming photons.

Increasing the interpillar gap beyond this value will although allow more incoming photons to get trapped inside the nanopillar array however, this excess gap would minimize the amount of their multiple internal reflections as shown in Fig. 4(d). All the five incoming photons have been trapped within the nanopillar gap with three, four, five and five internal reflections respectively. These reduced reflections would decrease the light reabsorption probability which would further minimize the overall photoresponsivity. Also with such a large interpillar gap there would be a decrement in the required number of nanopillars that could be deployed over the front detector's surface for optimum detector's efficiency.

Therefore, in order to achieve maximum photodetector's performance Eqn. (10) provides the optimum interpillar spacing. This optimum spacing could calculate the required number of the proposed half octagonal cut based hexagonal nanopillars to be deployed over the detector's front surface area to achieve maximum detector's efficiency.

The total number of nanopillar tiers that could be inlayed over ' $a$ um x $a$ um' surface area could be deduced as [20],

$$
n_{\text {tier }}=\frac{y-D}{2(D+d)}
$$

Here, ' $D=[(1+\sqrt{ } 2) l / 2]$ ' is the diameter of the hexagonal nanopillar and in terms of a very important parameter i.e, nanopillar filling ratio $(f=d / p)$ it could be reduced as; 


$$
\begin{aligned}
& f=\frac{d}{P} \\
& \Rightarrow f=\frac{d}{d+D} \\
& \Rightarrow D=\frac{f d}{1-f}
\end{aligned}
$$

Putting this value in Eqn. (11) we get,

$$
\begin{aligned}
& n_{\text {tier }}=\frac{y-\left[\frac{f d}{1-f}\right]}{2\left[\frac{f d}{1-f}+d\right]} \\
& \Rightarrow n_{\text {tier }}=\frac{(1-f) y-f d}{2[f d+(1-f) d]} \\
& \Rightarrow n_{\text {tier }}=\frac{(1-f) y-f d}{2 d} \\
& \Rightarrow n_{\text {tier }}=\frac{(1-f) y}{2 d}-\frac{f}{2} \\
& \Rightarrow n_{\text {tier }}=\frac{1}{2}\left[\frac{(1-f) y}{d}-f\right]
\end{aligned}
$$

Now with this nanopillar tiers the total number of required ' $N_{p}$ ' could be deduced from equation as,

$$
\begin{aligned}
& N_{p}\left(x_{n_{\text {tier }}} \times y_{n_{\text {tier }}}\right)=1+3 n_{\text {tier }}\left(n_{\text {tier }}+1\right)+4\left(n_{\text {tier }}-1\right) \\
& \Rightarrow N_{p}\left(x_{n_{\text {tier }}} \times y_{n_{\text {tier }}}\right)=1+3\left(n_{\text {tier }}\right)^{2}+7 n_{\text {tier }}-4 \\
& \Rightarrow N_{p}\left(x_{n_{\text {tier }}} \times y_{n_{\text {tier }}}\right)=n_{\text {tier }}\left[3 n_{\text {tier }}+7\right]-3
\end{aligned}
$$

Putting the value of ' $n$ ' in this equation we get,

$$
N_{p}\left(x_{n_{\text {tier }}} \times y_{n_{\text {tier }}}\right)=\left\{\frac{1}{2}\left[\frac{(1-f) y}{d}-f\right]\left[\frac{3}{2}\left[\frac{(1-f) y}{d}-f\right]+7\right]-3\right\}
$$

Now, putting the value of 'd' from Eqn. (10) we get,

$$
N_{p}\left(x_{n_{\text {ier }}} \times y_{n_{\text {ier }}}\right)=\frac{1}{2}\left[\frac{y(1-f) \tan \left(90^{\circ}-\theta_{i}\right)}{2\left(h-l \cos \theta_{t 1}\right)-l \sin \theta_{t 1} \tan \left(90^{\circ}-\theta_{i}\right)}-f\right]\left\{\frac{3}{2}\left[\frac{y(1-f) \tan \left(90^{\circ}-\theta_{i}\right)}{2\left(h-l \cos \theta_{t 1}\right)-l \sin \theta_{t 1} \tan \left(90^{\circ}-\theta_{i}\right)}-f\right]+7\right\}-3
$$

The photodetector performance is greatly impacted by the fresnel reflection losses $(\mathrm{R})$ that have to be reduced by enhancing the optical path length. The light reflectance relation [21], considering zero transmission losses may be written as;

$$
R(\lambda)+A((\lambda))=1
$$


Where, 'A' is the total light absorbance of the photodetector material.

With ' $N_{p h}$ ' number of proposed nanopillar deployment the overall reduced light reflectance(R) obtained for the ' $N_{p h}$ ' number of incoming photons trapped within the detector could be deduced as;

$$
\begin{gathered}
R=m\left\{R_{s}-\left[R_{s} \times R_{p 1}\left[1+R_{p 2}+R_{p 2} R_{p 3}\left[1+R_{p 4}+R_{p 4} R_{p 1}+R_{p 4} R_{p 1} R_{p 2} \ldots \ldots \ldots\right]\right]\right]\right\}-\left(N_{p h}-m\right) \\
\left\{R_{p 4}-\left[R_{p 4} \times R_{p 1}\left[1+R_{s}+R_{s} R_{p 2}\left[1+R_{p 3}+R_{p 3} R_{p 1}+R_{p 3} R_{p 1} R_{p 2} \ldots \ldots . .\right]\right]\right]\right\}
\end{gathered}
$$

Here, ' $m$ ' represents the number of photons striking the detector's planar surface directly prior to hitting at the proposed nanopillar interface out of total ' $N$ ph' number of incoming photons. Also,

$$
\begin{aligned}
& R_{s}=\frac{n_{1} \cos \theta_{i}-n_{2} \cos \theta_{t}}{n_{1} \cos \theta_{i}+n_{2} \cos \theta_{t}}, \quad R_{p 1}=\frac{n_{1} \cos \theta_{p 1}-n_{e f f} \cos \theta_{t p 1}}{n_{1} \cos \theta_{p 1}+n_{e f f} \cos \theta_{t p 1}} \\
& R_{p 2}=\frac{n_{1} \cos \theta_{p 2}-n_{e f f} \cos \theta_{t p 2}}{n_{1} \cos \theta_{p 2}+n_{e f f} \cos \theta_{t p 2}}, R_{p 3}=\frac{n_{1} \cos \theta_{p 3}-n_{e f f} \cos \theta_{t p 3}}{n_{1} \cos \theta_{p 3}+n_{e f f} \cos \theta_{t p 3}} \\
& R_{p 4}=\frac{n_{1} \cos \theta_{p 4}-n_{e f f} \cos \theta_{t p 4}}{n_{1} \cos \theta_{p 4}+n_{e f f} \cos \theta_{t p 4}}
\end{aligned}
$$

' $\theta_{t}$ ' is the transmission angle of the incoming photon through the detector's planar surface, ' $\theta_{t p 1}=\theta_{t p 2}=\theta_{t p 3}=\theta_{t p 4}$ ' are the photon transmission angles within the device through the proposed nanopillar interface and ' $n_{\text {eff' }}$ ' is the effective refractive index of the nanopillar which is given as [22],

$$
n_{\text {eff }}=n_{1}(1-f)+n_{\text {GaAs }} f
$$

Now, from Eqn. (13), 


$$
\begin{aligned}
& N_{p}\left(x_{n_{\text {tier }}} \times y_{n_{\text {tier }}}\right)=\frac{1}{2}\left\{\left[\frac{(1-f) y}{d}-f\right]\left[\frac{3}{2}\left[\frac{(1-f) y}{d}-f\right]+7\right]\right\}-3 \\
& \Rightarrow N_{p}\left(x_{n_{\text {tier }}} \times y_{n_{\text {tier }}}\right)=\frac{1}{2 d}\{[(1-f) y-d f][3(y-f y-d f+14 d)]\}-3 \\
& \Rightarrow N_{p}\left(x_{n_{\text {tier }}} \times y_{n_{\text {tier }}}\right)=\frac{1}{4 d^{2}}\left\{\begin{array}{l}
y[3 y-3 f y-3 d f+42 d]-f y[3 y-3 f y-3 d f+42 d] \\
d f[3 y-3 f y-3 d f+42 d]
\end{array}\right\}-3 \\
& \Rightarrow N_{p}\left(x_{n_{\text {tier }}} \times y_{n_{\text {tier }}}\right)=\frac{1}{4 d^{2}}\left\{\begin{array}{l}
3 y^{2}-3 f y^{2}-3 d f y+42 d y-3 f y^{2}+3 f^{2} y^{2}+3 d f^{2} y- \\
42 d f y-3 d f+3 d f^{2} y+3 d^{2} f^{2}-42 d^{2} f
\end{array}\right\}-3 \\
& \Rightarrow N_{p}\left(x_{n_{\text {tier }}} \times y_{n_{\text {tier }}}\right)=\frac{1}{4 d^{2}}\left\{\begin{array}{l}
3 y^{2}-6 f y^{2}-45 d f y+42 d y+3 f^{2} y^{2}+6 d f^{2} y-3 d f y+ \\
3 d^{2} f^{2}-42 d^{2} f
\end{array}\right\}-3 \\
& \Rightarrow N_{p}\left(x_{n_{\text {tier }}} \times y_{n_{\text {tier }}}\right)=\frac{3 y^{2}}{4 d^{2}}-\frac{6 f y^{2}}{4 d^{2}}-\frac{45 f y}{4 d}+\frac{42 y}{4 d}+\frac{3 f^{2} y^{2}}{4 d^{2}}+\frac{6 f^{2} y}{4 d}-\frac{3 f y}{d}+\frac{3 f^{2}}{4}-\frac{42 f^{2}}{4} \\
& -3 \\
& \Rightarrow N_{p}\left(x_{n_{\text {tier }}} \times y_{n_{\text {tier }}}\right)=f^{2}\left[\frac{3 y^{2}}{4 d^{2}}+\frac{6 y}{4 d}-\frac{42}{4}+\frac{3}{4}\right]+f\left[-\frac{6 y^{2}}{4 d^{2}}-\frac{45 y}{4 d}-\frac{3 y}{4 d}\right]+\frac{3 y^{2}}{4}-3 \\
& \Rightarrow N_{p}\left(x_{n_{\text {tier }}} \times y_{n_{\text {tier }}}\right)=f^{2}\left[\frac{3 y^{2}}{4 d^{2}}+\frac{6 y}{4 d}-\frac{39}{4}\right]+f\left[-\frac{6 y^{2}}{4 d^{2}}-\frac{48 y}{4 d}\right]+\frac{3 y^{2}}{4}-3 \\
& \Rightarrow f=\frac{\left[\frac{6 y^{2}}{4 d^{2}}+\frac{48 y}{4 d}\right]-\sqrt{\left[-\frac{6 y^{2}}{4 d^{2}}-\frac{48 y}{4 d}\right]^{2}-4\left[\frac{3 y^{2}}{4 d^{2}}+\frac{6 y}{4 d}-\frac{39}{4}\right]\left[\frac{3 y^{2}}{4}-3-N_{p}\right]}}{2\left[\frac{3 y^{2}}{4 d^{2}}+\frac{6 y}{4 d}-\frac{39}{4}\right]} \\
& \Rightarrow f=\frac{\left[\frac{6 y^{2}}{4 d^{2}}+\frac{48 y}{4 d}\right]-\sqrt{\left[-\frac{6 y^{2}}{4 d^{2}}-\frac{48 y}{4 d}\right]^{2}-\left[\frac{12 y^{2}}{4 d^{2}}+\frac{24 y}{4 d}-\frac{156}{4}\right]\left[\frac{3 y^{2}}{4}-3-N_{p}\right]}}{2\left[\frac{3 y^{2}}{4 d^{2}}+\frac{6 y}{4 d}-\frac{39}{4}\right]} \\
& \Rightarrow f=\frac{\left[\frac{6 y^{2}}{4 d^{2}}+\frac{48 y}{4 d}\right]-\sqrt{\left[\frac{-6 y^{2}-48 y d}{4 d^{2}}\right]^{2}-\left[\frac{12 y^{2}+24 y d-156 d^{2}}{4 d^{2}}\right]\left[\frac{3 d^{2} y^{2}-12 d^{2}-N_{p} d^{2}}{4 d^{2}}\right]}}{2\left[\frac{3 y^{2}+6 y d-39 d^{2}}{4 d^{2}}\right]} \\
& \Rightarrow f=\frac{\frac{1}{4 d^{2}}\left[6 y^{2}+48 y d\right]-\frac{1}{4 d^{2}} \sqrt{\left[-6 y^{2}-48 y d\right]^{2}-\left[12 y^{2}+24 y d-156 d^{2}\right]\left[3 d^{2} y^{2}-12 d^{2}-N_{p} d^{2}\right]}}{\frac{1}{4 d^{2}}\left[6 y^{2}+12 y d-78 d^{2}\right]} \\
& \Rightarrow f=\frac{\left[6 y^{2}+48 y d\right]-\sqrt{\left[-6 y^{2}-48 y d\right]^{2}-\left[12 y^{2}+24 y d-156 d^{2}\right]\left[3 d^{2} y^{2}-12 d^{2}-N_{p} d^{2}\right]}}{\left[6 y^{2}+12 y d-78 d^{2}\right]}
\end{aligned}
$$


Putting this value of ' $f$ ' in eqn. (19) we could calculate the effective refractive index of the proposed nanopillar array.

\section{Results and Discussion}

This section comprises of the graphical investigation of the effect of varying structural parameters of the proposed nanopillar array model over the photodetector's performance metrics. For the ease of the simulation convenience a bunch of seven incoming photons at $40^{\circ}$ planar incident angle emitted from $2 \mathrm{~mm}$ diameter GaAs source are considered to get trapped inside the proposed nanopillar array deployed over 5um x 5um detector's front surface area. Each of the side length of the half octagonal cut has been considered to be o 0.1 um in our analysis. The incident photons after being reflected from the planar detector's front surface undergoes maximum number of internal reflections within the nanopillar interspacing after hitting the various upper and lower nanopillar interfaces. This multiple reflection phenomenon boost up the reabsorption probability of the otherwise lost photons to a significant level. Figure 5 depicts the variation of the incoming photon absorbance with light incident angles at fixed upper tilt angles ' $\theta_{\mathrm{t} 1}$ '. As could be observed from the fig, with increasing light incident angle there is an increment in the total light absorbance. The main attributing factor being the increased number of the total increased internal reflections within the array due to smaller striking angles at the nanopillar interfaces. However, for a $0.4 \mathrm{um}$ high nanopillar array increasing the incident angles beyond $50^{\circ}$ incident angle would greatly enhance the interpillar spacing ' $d$ '. With such a large gap the reflected ray of the incoming photon could not reach the adjacent nanopillar interface. This could reduce the enough required striking points absorption reducing the reabsorption probability for optimum light. A maximum absorption of 0.999 absorbance is achieved at $50^{\circ}$ incident angle with a half octagonal cut of $70^{\circ}$ upper tilt.

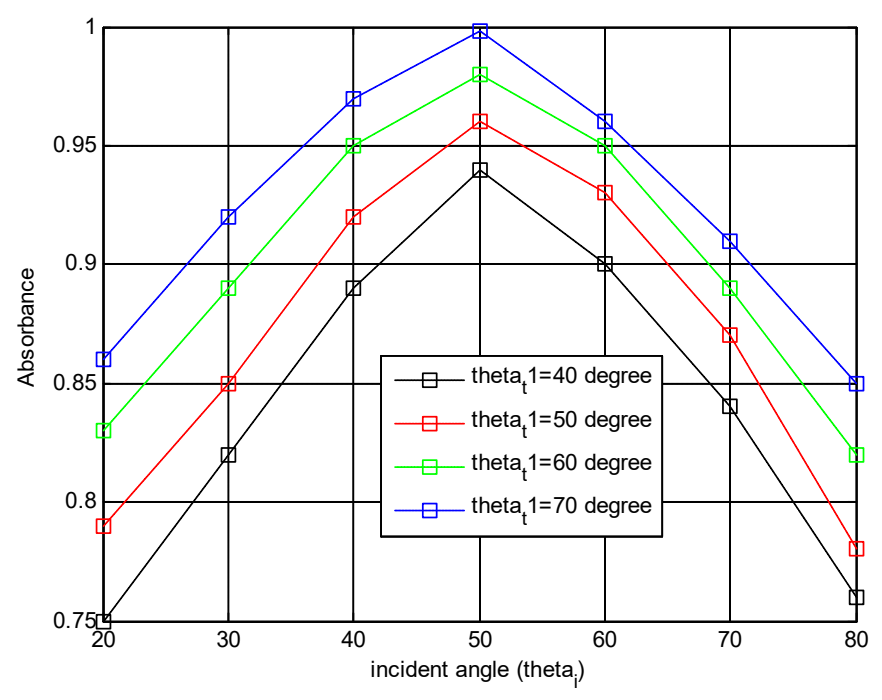

Fig. 5. Variation of light absorption with light incident angle at fixed upper tilted angle $\left(\theta_{t l}\right)$ from $40^{\circ}$ to $70^{\circ}$

Figure 6 represents the varying light absorbance plot with increasing half octagonal cut upper tilted angle at fixed center bending angles ' $\theta_{\mathrm{B}}$ '. With increasing upper tilting of the proposed model there is a greater chance of the reflected portion of the incoming photon to revert back towards the front detector's planar surface for reabsorption. Even after reflection from this point at the planar surface the secondary reflected ray undergoes even further maximum internal reflections within the nanopillar array. With increased centre bending angle there is a gradual sharp formation of the half octagonal cut over the nanopillar array. This cut will provide four nanopillar interfaces which will tremendously aid towards multiple internal reflections mitigating the Fresnel reflection losses to a minimal level. However, enhancing the bending angle beyond a certain point $\left(170^{\circ}\right)$ would reduce the overall light absorbance. This is due to the fact that increased bending angle would prohibit the incoming photon to revert back towards the planar detector's surface for further reabsorption. Instead the reflected ray would strike the upward cut of the adjacent nanopillar which would reduce the amount of internal 
reflection phenomenon ultimately not contributing much towards reduced Fresnel reflection losses. The $40^{0}$ incident photons getting trapped inside the $0.4 \mathrm{um}$ high nanopillar array would exhibit 0.999 absorbance with a $150^{\circ}$ centre bending angle at $60^{\circ}$ upper tilted angle.

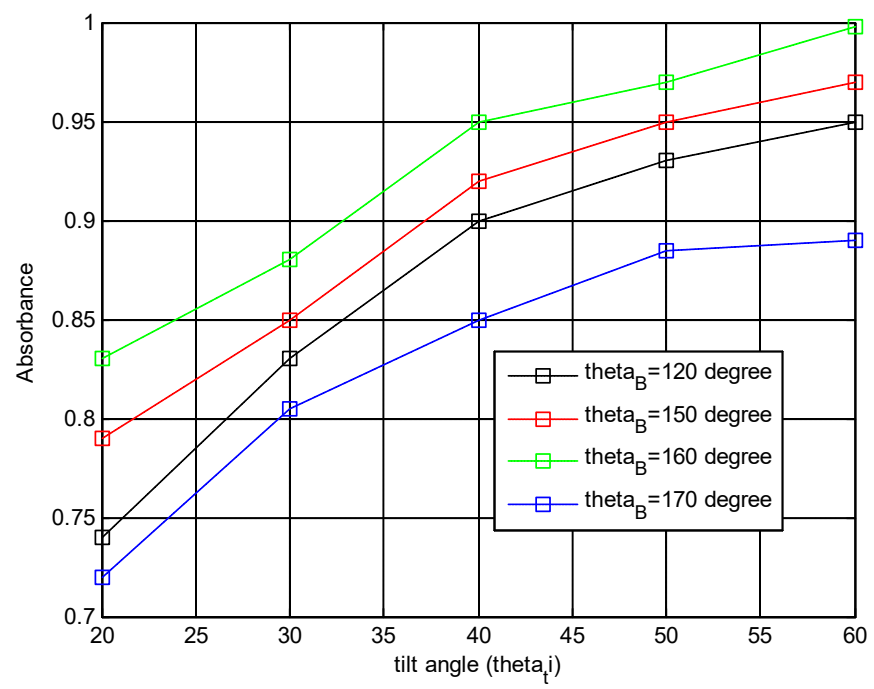

Fig. 6. Variation of light absorption with nanopillar upper octagonal cutting angle $\left(\theta_{t I}\right)$ at centre bending angles $\left(\theta_{B}\right)$ from $120^{\circ}$ to $170^{\circ}$ for ${ }^{~} 40^{0}$ 'light incident angle

The variation of light absorbance with increasing upper striking angle $\left(\theta_{\mathrm{p} 1}\right)$ has been provided in Fig.7. As could be observed from the simulative plot, with increasing upper striking angle there is an upsurge in the overall light absorbance. This is due to the fact that with increasing upper striking angle the secondary reflected ray from this point again hits back at the second lower interface of the nanopillar cut with a wider hitting angle. This large incident angle would aid the incoming photon to revert back at the detector's front surface and thus it remained trapped inside the proposed nanopillar array for optimum reabsorption probability. However, enhancing the striking angle beyond $\left(\theta_{\mathrm{B} 1}-90^{\circ}\right)$ would either allow the secondary reflected ray from this point to hit the second upper interface or the upper lower interface with smaller striking angle. Due to this smaller striking angle the incoming photon would undergo fewer internal reflections before finally getting lost to air. Thus, the reabsorption probability of the photon would reduce to a minimal level. The incoming photon attains a maximum absorbance of 0.999 with $70^{\circ}$ upper striking angle at $160^{\circ}$ upper bending angle.

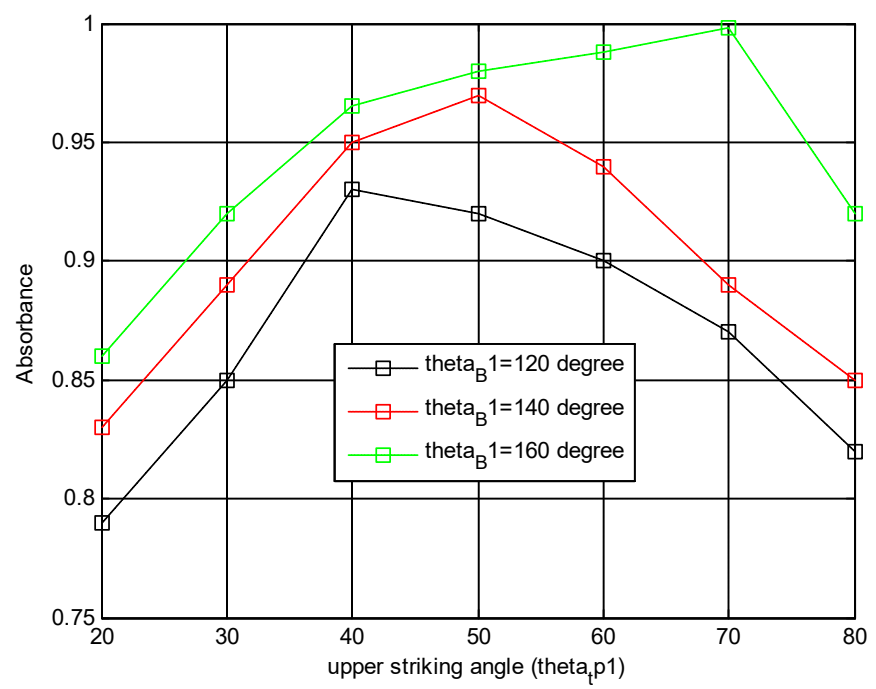


Fig. 7. Variation of light absorbance with nanopillar upper cut interface striking angle $\left(\theta_{p l}\right)$ at fixed upper octagonal bending angles $\left(\theta_{B I}\right) 120^{\circ}, 140^{\circ}$ and $160^{\circ}$ for ' $40^{\circ}$ ' light incident angle

Figure 8 represents the total number of proposed nanopillars of fixed height $0.2 \mathrm{um}, 0.4 \mathrm{um}$ and $0.5 \mathrm{um}$ with an appropriate interpillar spacing to be deployed over 5 um x 5 um detector's front surface area based on the photon incident angle $\left(\theta_{i}\right)$. As could be observed from the figure, with increasing light incident angle there is an overall reduction of the total number of proposed nanopillars that could be deployed over 5 um $\mathrm{x} 5$ um surface area. This is due to the increased interpillar spacing required for wider incident angled photons required for optimum light absorbance. This large interpillar gap would not allow accommodating the required proposed nanopillars of fixed height. For longer nanopillars deploying a fewer number of them would be enough to provide the maximum absorbance as they will provide the maximum number of internal reflections to the incoming photons. For smaller nanopillars comparatively a larger number is required for attaining the maximum light absorbance.A total of maximum 90 number of 0.2 um longer nanopillars are required to be deployed over 5um x 5um front detector's surface area with 0.04um interpillar gap for a $10^{0}$ light incident angle.

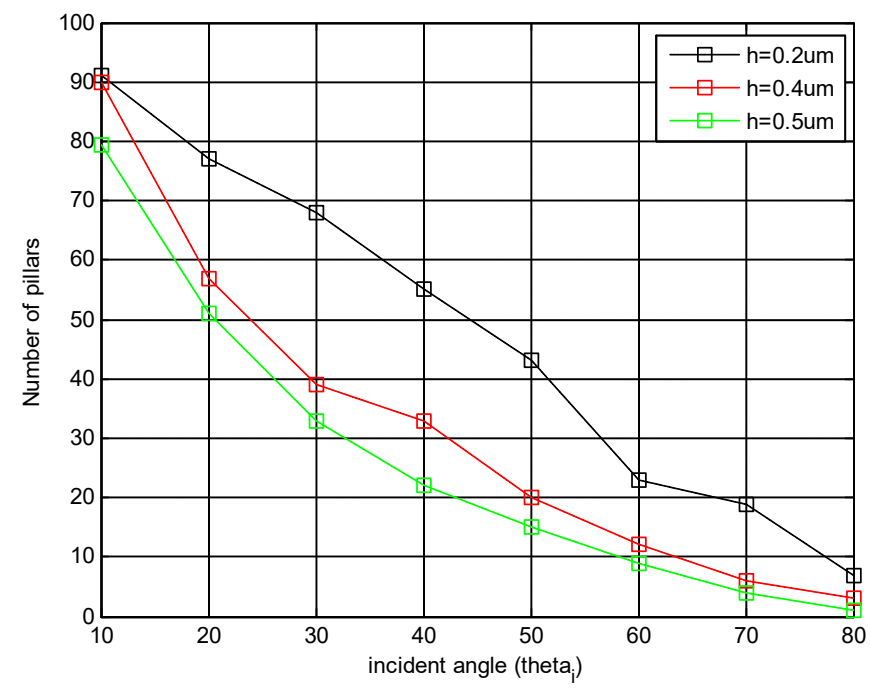

Fig. 8. Total number of half octagonal cut based hexagonal nanopillars of varying height from $0.2 \mathrm{um}$ to $0.5 \mathrm{um}$ deployed over '5um x 5um' detector's front surface w.r.t incoming light incident angle $\left(\theta_{i}\right)$

The variation of total light absorbance of $40^{\circ}$ incident angled photons with increasing nanopillar height from 0.2 um to $1.6 \mathrm{um}$ with fixed filling ratios from 0.2 to 0.4 has been demonstrated in Fig.9. As could be demonstrated from the figure, for the proposed nanopillar array with fixed interpillar gapthe total light absorbance increases with increasing pillar height as the allowed number of multiple internal reflections faced by the incoming photon enhances which boost up the reabsorption probability. However, increasing the pillar height beyond the range specified by the fixed interpillar gap would reduce the light absorbance owing to the nanopillar shadowing effect. This effect will prohibit the required number of incoming photons to get trap inside the array and instead reflect them directly to get lost in air. Thus, there will be a lesser effect on minimizing the Fresnel reflectance. As could be evaluated from the graphical plot, for higher filling ratios beyond a certain limit depending on the nanopillar diameter, pitch length as well as angle of light incidence the total light absorbance decreases. This is due to the reduced number of adequate proposed nanopillar deployment over the detector's surface that could provide the optimum light absorbance. A maximum of 0.999 absorbance has been attained for a $40^{\circ}$ incident photon ray trapped within a $1.1 \mathrm{um}$ high proposed nanopillar array with 0.3 filling ratio. 


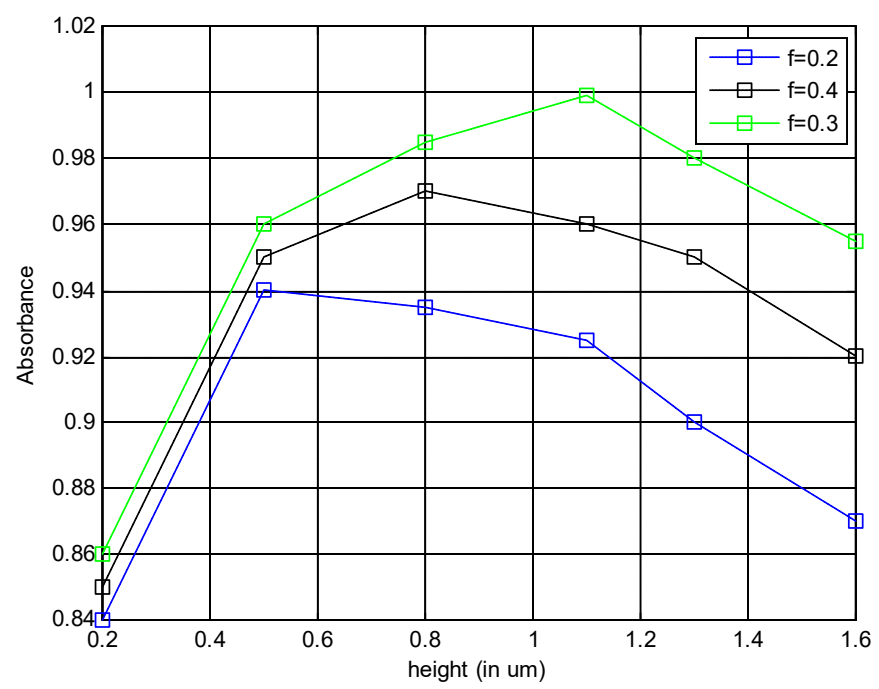

Fig. 9. Light absorbance variation obtained within the nanopillar array with increasing pillar height from $0.2 \mathrm{um}$ to 1.6 um at fixed filling ratio from 0.2 to 0.4

The overall reflectance of the $40^{\circ}$ incident incoming photons trapped inside the nanopillar array w.r.t the internanopillar spacing is depicted in Fig.10. As the figure shows, with an increasing interpillar spacing a maximum number of incoming photons could be trapped within the array so as to reduce the light reflectance to a minimal level. However, beyond a certain limit, enhancing the interpillar spacing would not assist in reflectance minimization as the incoming photons would not face any further multiple internal reflections within the array as the secondary reflected rays couldn't reach the adjacent nanopillar for hitting and getting reabsorbed. Thus, without much aiding towards the light absorbance the incoming would directly get lost to air. The plot shows a minimum reflectance of $0.1 \%$ obtained within a $1.4 \mathrm{um}$ high nanopillar array with $1 \mathrm{um}$ interpillar gap at lum operating wavelength.

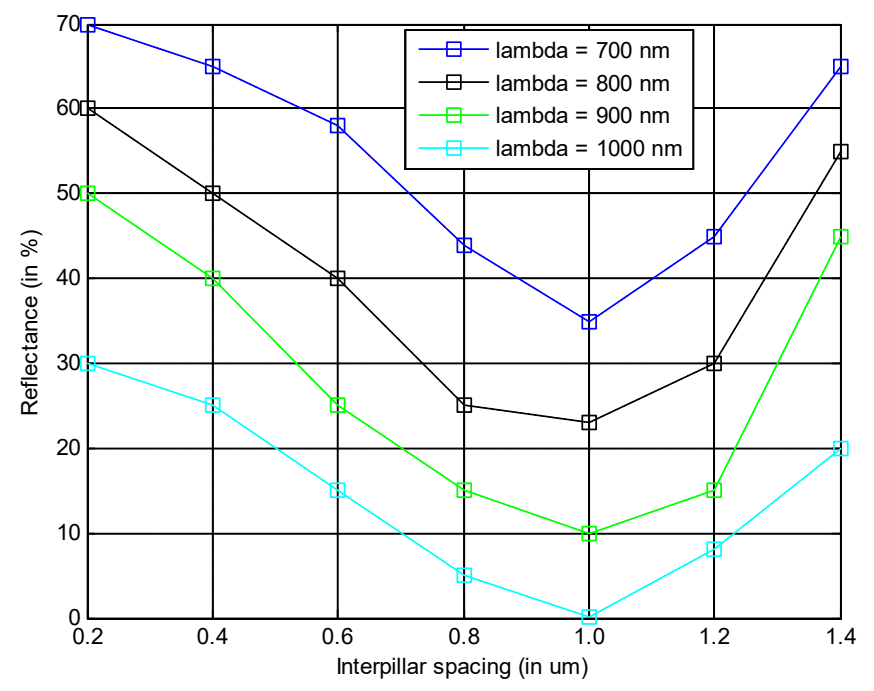

Fig. 10. Total light reflectance of $40^{\circ}$ incident photons with varying interpillar spacing at different operating wavelength from $0.7 \mathrm{um}$ to $1 \mathrm{um}$

Figure 11 demonstrates the effective refractive index minimization obtained with the deployment of the proposed nanopillar array with an increasing light incident angle $\left(\theta_{i}\right)$ at different nnanopillar length. As could be observed from the plot,with an increased photon incident angle upto $80^{\circ}$ the semiconductor-air mismatch 
reduces towards a lowest to 0.2 which reduces the light reflectance to an optimal level at $0.5 \mathrm{um}$ nanopillar height. This is due to the reduced filling ratio covering the detector's surface for maximum absorption at larger incident angles as well as nanopillar length.

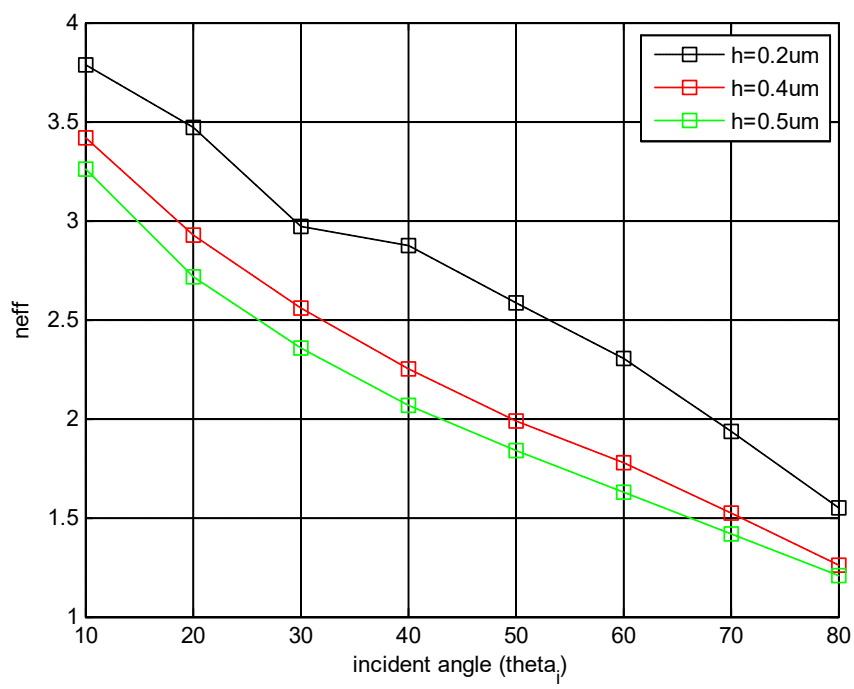

Fig. 11. Effective refractive index variation of the proposed nanopillar array according to the light incident angle at fixed nanopillar length from $0.2 \mathrm{um}$ to $0.5 \mathrm{um}$

Figure 12 and 13 compares the photoresponse characteristics curves of the proposed 0.4 um high periodic half octagonal cut based InGaAs photodetector to that of our previously optimised model of InGaAs hexagonal based nanopillar array and planardetector's surface in terms of responsivity and EQE plot. Enhanced carrier absorption with the proposed nanopillarray structure would result in higher electron-hole pair generation that leads towards obtaining responsivity of $0.99 \mathrm{~A} / \mathrm{W}$ at $1 \mathrm{~mW}$ input optical power in comparison to the $0.93 \mathrm{~A} / \mathrm{W}$ attained with the hexagonal based nanopillar array [22]. The external quantum efficiency (EQE) performance of $86 \%$ also has a boost of approximately $11 \%$ in comparison to that of $75.5 \% \mathrm{EQE}$ obtained with the hexagonal nanopillar array with a narrow 0.5 um depletion width.

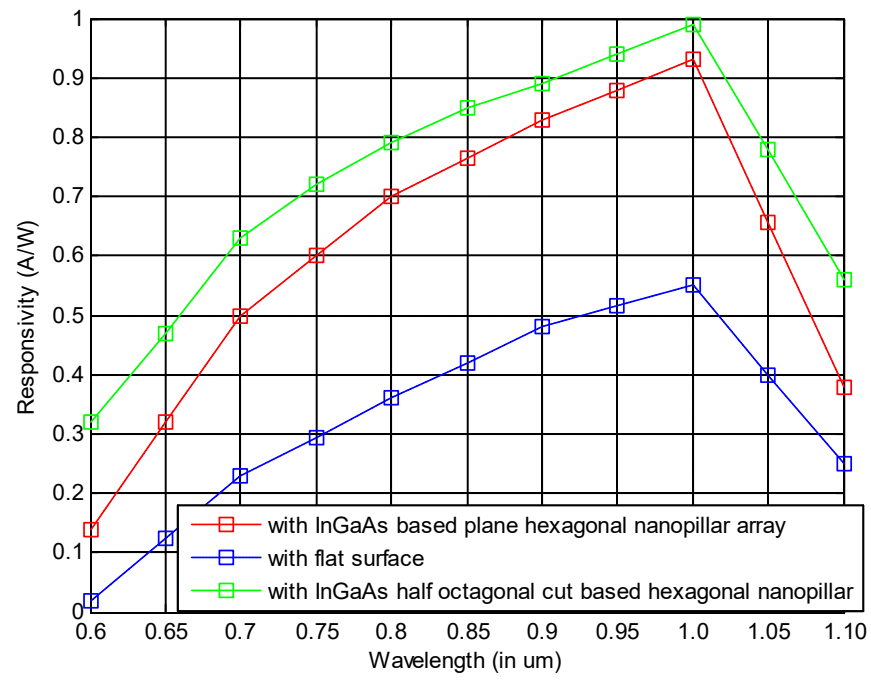

Fig. 12. Photoresponse characteristic comparison of the device with proposed half octagonal cut based textured hexagonal nanopillar array surface, planar hexagonal based nanopillar array and flat GaAs surface for various wavelength range with parameters: $h=0.4 u m, f=0.3$ and depletion width $\left(w_{d}\right)=0.5 u m$ 


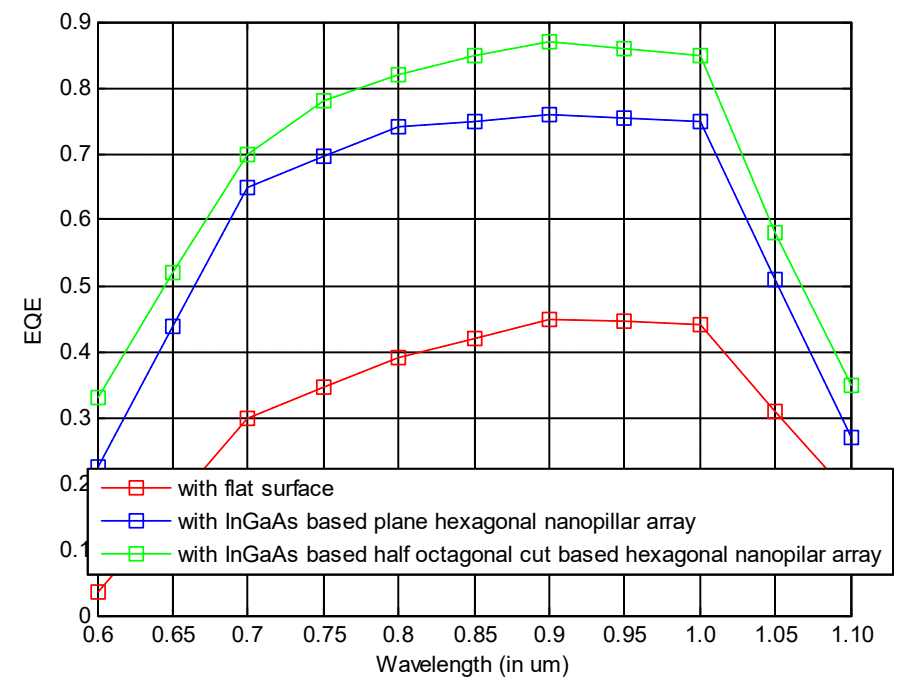

Fig. 13. $\mathrm{EQE}(\eta)$ of the photodetector obtained with proposed InGaAs based nanopillar array structure $(h=0.4 u m, f=0.3 u m, d=0.1 u m)$, planar InGaAs based anopillar array structure and flat surface $\left(w_{s}=0.5 u m\right)$ for a wavelength range of $0.6 \mathrm{um}$ to $1.1 \mathrm{um}$ with a $0.5 \mathrm{um}$ depletion width

After optimizing the proposed half octagonal cut based textured nanopillar array model in terms of structural parameters including upper and lower nanopillar angular tilt,three upper,lower and centre bending angles,nanopillar four interfaces striking angles along with material and composition properties including filling ratio,effective refractive index and different device parameters are fitted to do the detector's performance analysis. The proposed nanopillar structure would greatly contribute towards light reflectance mitigation mechanism and thus reduced the incoming light information loss. Therefore, this structure could be adopted in various optical applications including photodetection, solarcells, bio-sensing, imaging etc as well as on several opto wireless solutions.

In order to do the comparative study of the proposed model in terms of absorption, reflectivity and responsivity three different kinds of published literatures are considered. In Table 1, it has been evaluated that from $400 \mathrm{~nm}$ to $800 \mathrm{~nm}$ wavelength range, the reflectivity obtained within a single interpillar spacing of the two adjacent faces of proposed model is lesser then that of the two different tapered nanostructured based NP arrays proposed by R. Santania et al.[12], our previous models [19] and [20]. Table 2 compares the measured absorption spectra for the wavelength range from $1040 \mathrm{~nm}$ to $2300 \mathrm{~nm}$ obtained from the work by Wook-Jae Lee et.al [13], our previous hexagonal based nanopillar array model [20], and our proposed half octagonal cut based textured model. As could be seen from the table, the light absorption values obtained with the proposed model are higher for the same operating wavelength range in comparison to the data provided in the existing literature From Table 3 it has been quite evident that the comparison of the photoresponsive characteristics as well as the measured reflectance spectra obtained as per the work done by T. Kan et al. [23],our previous model [19], previous InGaAs based plane hexagonal pillar [20] and the proposed model for different wavelength ranges. It has been observed from the comparison tables that the proposed half octagonal based nanopillar array for any material would provide a higher photon absorbance in comparison to previous nanopillar based models. Although there is a slight change obtained in photon absorption within the single interpillar spacing of the proposed in comparison to previous model [19] however the overall absorption of the incoming photons with the proposed model will be high as incoming photons will undergo more multiple internal reflections within six inter faces of the proposed hexagonal nanopillar due to the octagonal cut in comparison to only four inter faces of the pyramidal cut based nanopillar. For ' $p$ ' number of incoming photons trapped within a single interpillar spacing of two adjacent faces of the nanopillar structure, a single pyramidal cut nanopillar would grasp ' $4 p$ ' number of photons within its four interfacial spacing with four of the adjacent faces of the nearby nanopillars. On the other hand a hexagonal nanopillar structure could grasp ' $6 p$ ' number of photons within the intergap with 
six of adjacent faces of the nearby nanopillars. Multiple reflections within interpillar spacing from six adjacent faces of the nearby proposed nanopillars would boost up the photon absorption to an optimum level by increasing the light reabsorption probability.

Table 1. Photodetector performances comparison of the proposed half octagonal cut based hexagonal nanopillar array model with the GaAs material based various Nanopillar array structures over a detector's surface area of $100 \mathrm{um} \times 100 \mathrm{um}$ for $7.5^{\circ}$ photon incident angle

\begin{tabular}{|c|c|c|c|c|c|c|c|}
\hline \multirow[b]{2}{*}{ Models } & \multicolumn{6}{|c|}{ Parameters } & \\
\hline & Structure & Dimension & $\begin{array}{l}\text { Materia } \\
\text { Is }\end{array}$ & $\begin{array}{l}\text { Pitch } \\
(\mu \mathrm{m})\end{array}$ & $\begin{array}{l}\text { Wavelength } \\
(\lambda) \text { in } \mathbf{n m}\end{array}$ & Reflectivity & \\
\hline \multirow{10}{*}{ Model[12] } & \multirow{5}{*}{ Cone } & \multirow{5}{*}{$\begin{array}{l}\text { Height }=0.79 \mu \mathrm{m} \\
\text { Diameter }=0.45 \mu \mathrm{m}\end{array}$} & \multirow{10}{*}{ GaAs } & \multirow{10}{*}{0.5} & 400 & $\sim 2.5$ & \\
\hline & & & & & 500 & $\sim 3$ & \\
\hline & & & & & 600 & $\sim 2.5$ & \\
\hline & & & & & 700 & $\sim 2$ & \\
\hline & & & & & 800 & $\sim 1$ & \\
\hline & \multirow{5}{*}{ Frustum } & \multirow{5}{*}{$\begin{array}{l}\text { Height }=2.7 \mu \mathrm{m} \\
\text { Diameter: } \\
\text { Upper }=0.1 \mu \mathrm{m} \\
\text { Lower }=0.45 \mu \mathrm{m}\end{array}$} & & & 400 & $\sim 1.5$ & \\
\hline & & & & & 500 & $\sim 0.7$ & \\
\hline & & & & & 600 & $\sim 1.5$ & \\
\hline & & & & & 700 & $\sim 0.3$ & \\
\hline & & & & & 800 & $\sim 0.5$ & \\
\hline \multirow{5}{*}{$\begin{array}{c}\text { Our } \\
\text { previous } \\
\text { Model [19] }\end{array}$} & \multirow{5}{*}{$\begin{array}{l}\text { Pyramidal } \\
\text { Cut } \\
\text { Nanopillar }\end{array}$} & \multirow{5}{*}{$\begin{array}{l}\text { (a) } \text { Height }=0.79 \mu \mathrm{m} \\
\text { Width }=0.45 \mu \mathrm{m} \\
\text { (b) } \text { Height }=2.7 \mu \mathrm{m} \\
\text { Width }=0.45 \mu \mathrm{m}\end{array}$} & \multirow{5}{*}{ GaAs } & \multirow{5}{*}{$\begin{array}{c}0.672 \\
\text { and } \\
0.681\end{array}$} & 400 & 1.4 & \multirow{15}{*}{ 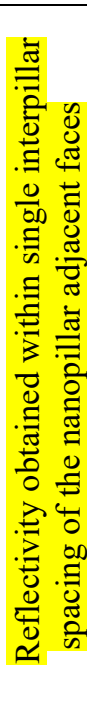 } \\
\hline & & & & & 500 & 0.1 & \\
\hline & & & & & 600 & 0.3 & \\
\hline & & & & & 700 & 0.24 & \\
\hline & & & & & 800 & 0.2 & \\
\hline \multirow{5}{*}{$\begin{array}{c}\text { Our } \\
\text { previous } \\
\text { Model [20] }\end{array}$} & \multirow{5}{*}{$\begin{array}{l}\text { Planar } \\
\text { Hexagonal } \\
\text { nanopillar } \\
\text { array }\end{array}$} & \multirow{5}{*}{$\begin{array}{l}\text { (a) } \text { Height }=0.79 \mu \mathrm{m} \\
\text { Width }=0.45 \mu \mathrm{m} \\
\text { (b) } \text { Height }=2.7 \mu \mathrm{m} \\
\text { Width }=0.45 \mu \mathrm{m}\end{array}$} & \multirow[t]{5}{*}{ GaAs } & \multirow{5}{*}{$\begin{array}{c}0.672 \\
\text { and } \\
0.681\end{array}$} & 400 & 0.8 & \\
\hline & & & & & 500 & 1.2 & \\
\hline & & & & & 600 & 0.8 & \\
\hline & & & & & 700 & 0.2 & \\
\hline & & & & & 800 & 0.4 & \\
\hline \multirow{5}{*}{$\begin{array}{l}\text { Proposed } \\
\text { Model }\end{array}$} & \multirow{5}{*}{$\begin{array}{c}\text { Half } \\
\text { Octagonal } \\
\text { cut based } \\
\text { hexagonal } \\
\text { nanopillar } \\
\text { array }\end{array}$} & \multirow{5}{*}{$\begin{array}{l}\text { (a) } \text { Height }=0.79 \mu \mathrm{m} \\
\text { Width }=0.45 \mu \mathrm{m} \\
\text { (b) } \text { Height }=2.7 \mu \mathrm{m} \\
\text { Width }=0.45 \mu \mathrm{m}\end{array}$} & \multirow{5}{*}{ GaAs } & \multirow{5}{*}{$\begin{array}{c}0.672 \\
\text { and } \\
0.681\end{array}$} & 400 & 0.08 & \\
\hline & & & & & 500 & 0.03 & \\
\hline & & & & & 600 & 0.09 & \\
\hline & & & & & 700 & 0.11 & \\
\hline & & & & & 800 & 0.08 & \\
\hline
\end{tabular}

Table 2. Absorption spectra comparative analysis of the proposed nanopillar array model with 1um high InAsSb based vertical NP structure array consisting of $0.18 \mathrm{um}$ wide Nanopillars mounted over a 50um x 50um detector's surface area for $16^{0}$ incident angle

\begin{tabular}{|c|c|c|c|c|c|}
\hline \multirow{3}{*}{ Models } & \multicolumn{5}{|c|}{ Parameters } \\
\cline { 2 - 6 } & Structure & Materials & Pitch $(\boldsymbol{\mu m})$ & $\begin{array}{c}\text { Wavelength } \\
(\boldsymbol{\lambda}) \text { in } \mathbf{~ n m}\end{array}$ & Absorbance \\
\hline \multirow{3}{*}{ Model[13] } & & & 1040 & $\sim 0.05$ \\
\cline { 5 - 6 } & Vertical & \multirow{2}{*}{ Au coated } & 0.9 & 1370 & $\sim 0.4$ \\
\cline { 4 - 6 } & & & & 1950 & $\sim 0.01$ \\
\hline
\end{tabular}




\begin{tabular}{|c|c|c|c|c|c|c|}
\hline & Nanopillar & InAsSb & & 2390 & $\sim 0.2$ & \\
\hline \multirow{4}{*}{$\begin{array}{c}\text { Our previous } \\
\text { Model [19] }\end{array}$} & \multirow{4}{*}{$\begin{array}{c}\text { Pyramidal Cut } \\
\text { vertical Nanopillar }\end{array}$} & \multirow{4}{*}{ InAsSb } & \multirow{4}{*}{0.75} & 1040 & 0.996 & \multirow{12}{*}{ 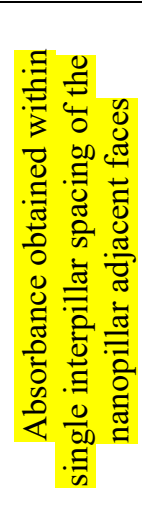 } \\
\hline & & & & 1370 & 0.989 & \\
\hline & & & & 1950 & 0.989 & \\
\hline & & & & 2390 & 0.995 & \\
\hline \multirow{4}{*}{\begin{tabular}{l}
\multicolumn{1}{c}{ Our } \\
previous \\
Model [20]
\end{tabular}} & \multirow{4}{*}{$\begin{array}{l}\text { Planar Hexagonal } \\
\text { nanopillar array }\end{array}$} & \multirow[t]{4}{*}{ InAsSb } & \multirow[t]{4}{*}{0.75} & 1040 & 0.956 & \\
\hline & & & & 1370 & 0.93 & \\
\hline & & & & 1950 & 0.937 & \\
\hline & & & & 2390 & 0.965 & \\
\hline \multirow{4}{*}{$\begin{array}{c}\text { Proposed } \\
\text { Model }\end{array}$} & \multirow{4}{*}{$\begin{array}{l}\text { Half Octagonal cut } \\
\text { based hexagonal } \\
\text { nanopillar array }\end{array}$} & \multirow{4}{*}{ InAsSb } & \multirow{4}{*}{0.75} & 1040 & 0.998 & \\
\hline & & & & 1370 & 0.999 & \\
\hline & & & & 1950 & 0.995 & \\
\hline & & & & 2390 & 0.99 & \\
\hline
\end{tabular}

Table 3. Comparative analysis of photo sensing performance of Si based vertical NP array structure consisting of $2.7 \mathrm{um}$ high and $0.2 \mathrm{um}$ wide nanopillars inlayed over a $100 \mathrm{~cm}$ x $100 \mathrm{~cm}$ detector's surface area for $8^{0}$ photon incident angle

\begin{tabular}{|c|c|c|c|c|c|c|c|}
\hline \multirow[b]{2}{*}{ Models } & \multicolumn{6}{|c|}{ Parameters } & \\
\hline & Structure & Materials & $\begin{array}{l}\text { Pitch } \\
(\mu \mathrm{m})\end{array}$ & $\begin{array}{l}\text { Wavelength } \\
(\lambda) \text { in nm }\end{array}$ & $\begin{array}{c}\text { Responsivity } \\
(\mathrm{mA} / \mathrm{W})\end{array}$ & $\begin{array}{c}\text { Reflectivity } \\
\text { (\%) }\end{array}$ & \\
\hline \multirow{5}{*}{ Model[23] } & \multirow{5}{*}{$\begin{array}{l}\text { Vertical } \\
\text { Nanopillar }\end{array}$} & \multirow{5}{*}{$\begin{array}{l}\text { Au coated } \\
\text { Si }\end{array}$} & \multirow{5}{*}{1} & 1.1 & 2.2 & 0.18 & \\
\hline & & & & 1.2 & 1 & 0.18 & \\
\hline & & & & 1.3 & 0.5 & 0.2 & \\
\hline & & & & 1.4 & 0.05 & 0.3 & \\
\hline & & & & 1.5 & 0.03 & 0.3 & \\
\hline \multirow{5}{*}{$\begin{array}{c}\text { Our previous } \\
\text { Model [19] }\end{array}$} & \multirow{5}{*}{$\begin{array}{l}\text { Pyramidal } \\
\text { Cut } \\
\text { vertical } \\
\text { Nanopillar }\end{array}$} & \multirow{5}{*}{$\mathrm{Si}$} & \multirow{5}{*}{0.95} & 1.1 & 2.65 & 0.01 & \multirow{15}{*}{ 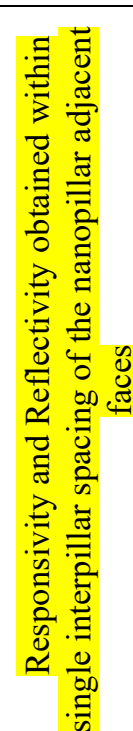 } \\
\hline & & & & 1.2 & 1.39 & 0.016 & \\
\hline & & & & 1.3 & 0.61 & 0.02 & \\
\hline & & & & 1.4 & 0.07 & 0.02 & \\
\hline & & & & 1.5 & 0.04 & 0.02 & \\
\hline \multirow{5}{*}{\begin{tabular}{c}
\multicolumn{1}{c}{ Our } \\
previous \\
Model [20]
\end{tabular}} & \multirow{5}{*}{$\begin{array}{c}\text { Planar } \\
\text { Hexagona } \\
1 \\
\text { nanopillar } \\
\text { array } \\
\end{array}$} & \multirow[t]{5}{*}{$\mathrm{Si}$} & \multirow[t]{5}{*}{0.95} & 1.1 & 3.4 & 0.1 & \\
\hline & & & & 1.2 & 1.7 & 0.08 & \\
\hline & & & & 1.3 & 0.8 & 0.1 & \\
\hline & & & & 1.4 & 1 & 0.15 & \\
\hline & & & & 1.5 & 0.08 & 0.12 & \\
\hline \multirow{5}{*}{$\begin{array}{c}\text { Proposed } \\
\text { Model }\end{array}$} & \multirow{5}{*}{$\begin{array}{c}\text { Half } \\
\text { Octagonal } \\
\text { cut based } \\
\text { hexagonal } \\
\text { nanopillar } \\
\text { array }\end{array}$} & \multirow{5}{*}{$\mathrm{Si}$} & \multirow{5}{*}{0.95} & 1.1 & 1.0 & 0.01 & \\
\hline & & & & 1.2 & 0.3 & 0.007 & \\
\hline & & & & 1.3 & 0.02 & 0.005 & \\
\hline & & & & 1.4 & 0.02 & 0.005 & \\
\hline & & & & 1.5 & 0.01 & 0.006 & \\
\hline
\end{tabular}

\section{Conclusion}

A periodically arranged GaAs material based half octagonal cut based textured nanopillar array model has been proposed to be inlayed over a photodetector's front detector's surface of same material in order to mitigate the Fresnel light reflectance losses caused due to the air-semiconductor refractive mismatch. This key factor that attributes towards the overall light reflectance mitigation is the multiple internal reflections phenomenon that took place within the proposed nanopillar array structure which boosts up the reabsorption probability 
significantly. This whole phenomenon is directly impacted by the structural parameters in terms of the upper and lower cutting angle of the proposed nanopillar structure along with its upper, centre and lower bending angles.Depending upon centre bending angle of the proposed nanopillar cut the incoming photon that get trapped within the nanopillar array undergoes maximum internal reflections minimizing the overall reflectance phenomenon. The proposed nanopillar array structure exhibits $86 \% \mathrm{EQE}$ with a photoresponsivity of $0.99 \mathrm{~A} / \mathrm{W}$ at 1 um operating wavelength with 0.5 um depletion width.

\section{References:}

1. Li, Chao and Huang, Weichun and Gao, Lingfeng and Wang, Huide and Hu, Lanping and Chen, Tingting and Zhang, Han, "Recent advances in solution-processed photodetectors based on inorganic and hybrid photo-active materials," Nanoscale, 12,4,2201-2227,2020.

2. Harry Efstathiadis and Pradeep Haldar and Nibir K. Dhar and Dennis L. PollaAshok K. Sood and John W. Zeller and Robert A. Richwine and Yash R. Puri, "SiGe Based Visible-NIR Photodetector Technology for Optoelectronic Applications,"Advances in Optical Fiber Technology,chapter-10,315361,2015 .

3. Santiago Royo and Maria Ballesta-Garcia, "An Overview of Lidar Imaging Systems for Autonomous vehicles,"Appl. Sci. 2019, 9(19), 4093.

4. Wai Son Ko, Indrasen Bhattacharya, Thai-Truong D.Tran, KarWei Ng, StephenAdair Gerke \& ConnieChang-Hasnain, "Ultrahigh Responsivity-Bandwidth Product in a Compact InP Nanopillar Phototransistor Directly Grown on Silicon,"Scientific Reports, 6:33368,1-11,2016.

5. Jiang Jing,Xu Zhida,Lin Jiahao,Gang Logan Liu, "Lithography-Free, Low-Cost Method for Improving Photodiode Performance by Etching Silicon Nanocones as Antireflection Layer,"Journal of Sensors, 1687-725X,2016.

6. Yu-Cheng Kao, Hao-Ming Chou, Shun-Chieh Hsu, Albert Lin, Chien-Chung Lin, Zun-Hao Shih, Chun-Ling Chang, Hwen-Fen Hong \& Ray-Hua Horng, "Performance comparison of III-V//Si and III-V//InGaAs multi-junction solar cells fabricated by the combination of mechanical stacking and wire bonding," Scientific Reports, volume 9, Article number: 4308, 2019.

7. Z. Mia and Y.-L., "Chang III-V compound semiconductor nanostructures on silicon: Epitaxial growth, properties, and applications in light emitting diodes and lasers," Journal of Nanophotonics, Vol. 3, 031602, 23 January 2009.

8. Kar Wei Ng, Thai-Truong D. Tran, Wai Son Ko, Roger Chen, Fanglu Lu and Connie J. ChangHasnain, "Single Crystalline InGaAsNanopillar Grown on Polysilicon with Dimensions beyond Substrate Grain Size Limit," NanoLett., vol.13, no.12, pp.5931-5937, 2013.

9. Wai Son Ko, "Photonic Integrated Circuits Using III-V Nanopillars Grown on Silicon,"Technical Report No. UCB/EECS-2016-172, December 1, 2016.

10. Kar Wei Ng and Constance Chang-Hasnain, "III-V Nanostructures on Dissimilar Substrates for Optoelectronic Applications," Technical Report No. UCB/EECS-2014-196, December 1, 2014.

11. Nazarenko, Maxim \&Sibirev, Nickolay\& Ng, Kar Wei \& Ren, Fan \& Son Ko, Wai\& G. Dubrovskii, Vladimir \& Chang-Hasnain, Connie, "Elastic energy relaxation and critical thickness for plastic deformation in the core-shell InGaAs/GaAsnanopillars," Journal of Applied Physics, 113, 2013.

12. R. Sanatinia, K. M. Awan, S. Naureen, N. Anttu, E. Ebraert, and S. Anand, "GaAsnanopillar arrays with suppressed broadband reflectance and high optical quality for photovoltaic applications," Opt. Mater. Express 2, 1671-1679, 2012.

13. Wook-Jae Lee, Pradeep Senanayake, Alan C. Farrell, Andrew Lin, Chung-Hong Hung, and Diana L. Huffaker, "High Quantum Efficiency Nanopillar Photodiodes Overcoming the Diffraction Limit of Light,"Nano Letters, 16 (1), 199-204, 2016.

14. Pradeep Senanayake, Chung-Hong Hung, Joshua Shapiro, Andrew Lin, Baolai Liang, Benjamin S. Williams, and D. L. Huffaker, "Surface Plasmon-Enhanced NanopillarPhotodetectors," Nano Letters, 11 (12), 5279-5283, 2011.

15. Pradeep Senanayake, Chung-Hong Hung, Alan Farrell, David A. Ramirez, Joshua Shapiro, Chi-Kang Li, Yuh-Renn Wu, Majeed M. Hayat, and Diana L. Huffaker, "Thin 3D Multiplication Regions in Plasmonically Enhanced Nanopillar Avalanche Detectors," Nano Letters, 12 (12), 6448-6452, 2012. 
16. Cai, Jinguang and Qi, Limin, "Recent advances in antireflective surfaces based on nanostructure arrays," Mater. Horiz., Volume 2, Number 1, pp. 37-53, 2015.

17. Diedenhofen, S. L., Vecchi, G. , Algra, R. E., Hartsuiker, A., Muskens, O. L., Immink, G. , Bakkers, E. P., Vos, W. L. and Rivas, J. G., "Broad-band and Omnidirectional Antireflection Coatings Based on Semiconductor Nanorods,” Adv. Mater., 21: 973-978, 2009.

18. Liang, D., Huo, Y., Kang, Y., Wang, K. X., Gu, A., Tan, M., Yu, Z., Li, S., Jia, J., Bao, X., Wang, S., Yao, Y., Wong, H. P., Fan, S., Cui, Y., and Harris, J. S., "Optical Absorption Enhancement: Optical Absorption Enhancement in Freestanding GaAs Thin Film Nanopyramid Arrays," Adv. Energy Mater., 2: 1150-1150, 2012.

19. SmritiBaruah, Santanu Maity, and Joyatri Bora, "High performance wide response GaAs based photodetector with nano texture on nanopillar arrays structure,"Microsyst. Tech.,26:2651-2660,2020.

20. SmritiBaruah, Santanu Maity, and Joyatri Bora, "Investigation and optimization of light trapping through hexagonal-shaped nanopillar (NP) array of Indium Gallium Arsenide material based photodetector,"Optical and Quantum Electronics, 52,1-17(380),2020.

21. Marco Volterrani,AlbertoMinelli, Monica Gaetani, Nicola Grossi., "Reflectance,absorbance and Transmittance spectra of bermudagrass and manaliagrassturfgrasscanocopies,"Ploss One., 12:1$13,2017$.

22. Guo H, Wen L, Li X, Zhao Z, Wang Y., "Analysis of optical absorption in GaAs nanowire arrays," Nanoscale Res Lett. 2011;6(1):617.

23. T. Kan, Y. Ajiki, K. Matsumoto and I. Shimoyama, "Si process compatible near-infrared photodetector using AU/SI nano-pillar array," 2016 IEEE 29th International Conference on Micro Electro Mechanical Systems (MEMS), Shanghai, 2016, pp. 624-627. 


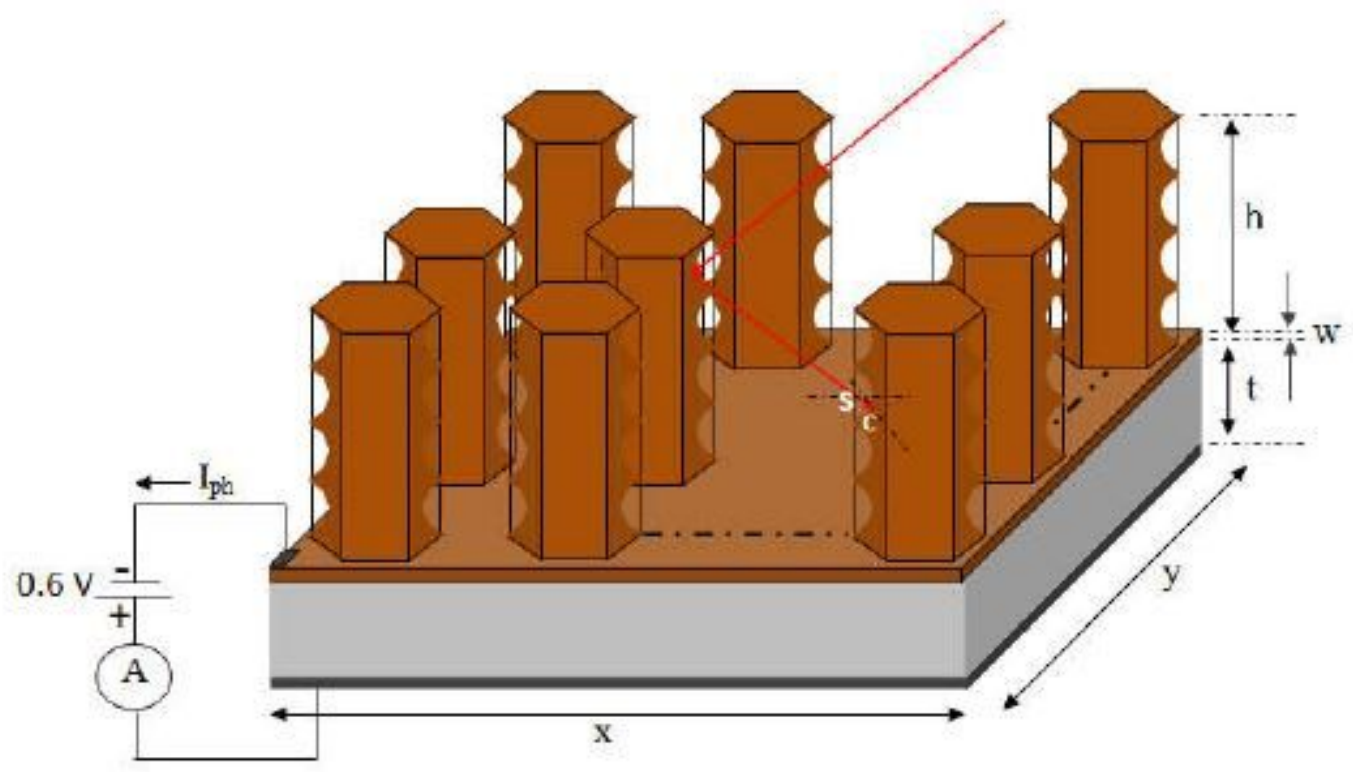

(a)

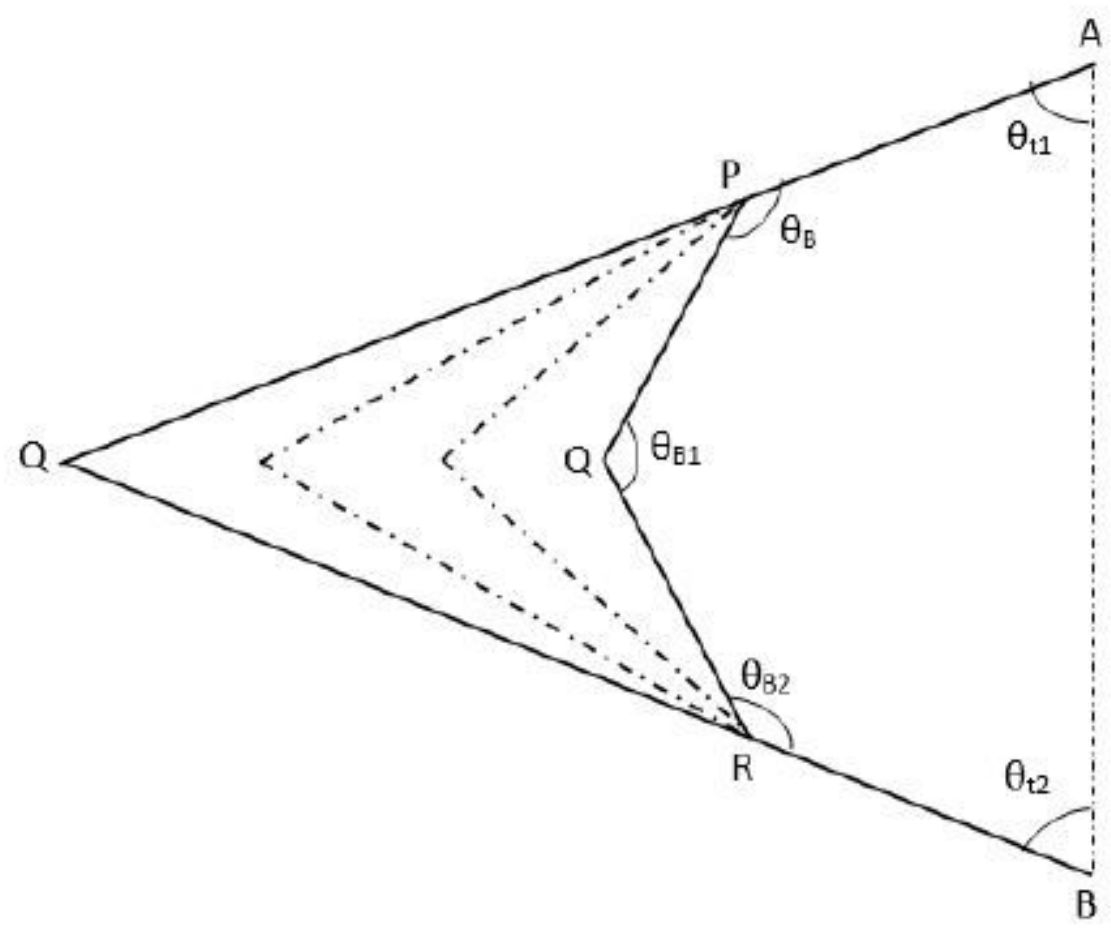

(b)

Figure 1

(a) Light interaction pattern within a proposed GaAs based half octagonal cut based nanotextured hexagonal-shaped nanopillar array photodetector surface structure, and (b) Schematic showing the half octagonal cut transformation from a pyramidal shaped cut. 


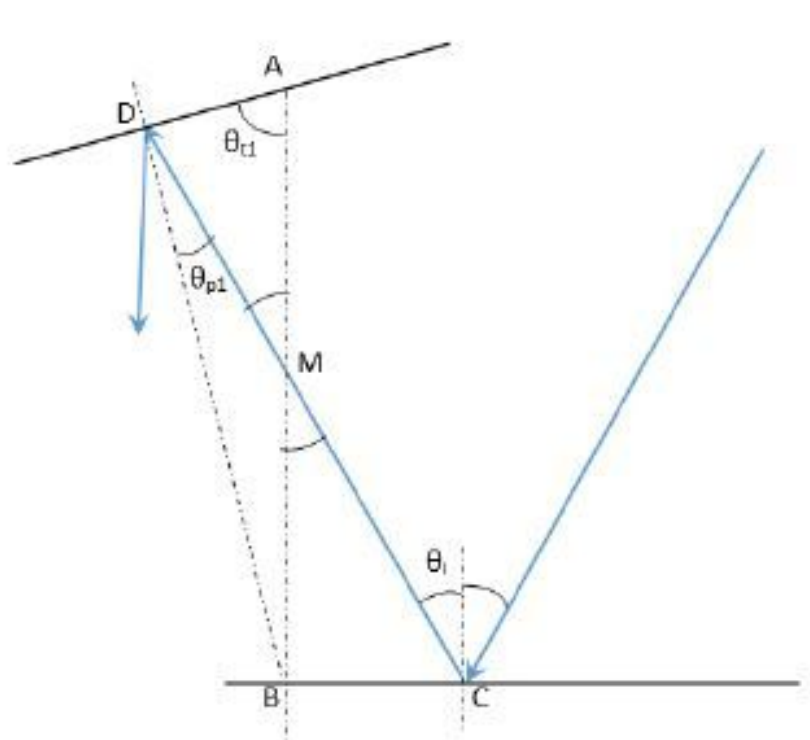

(a)

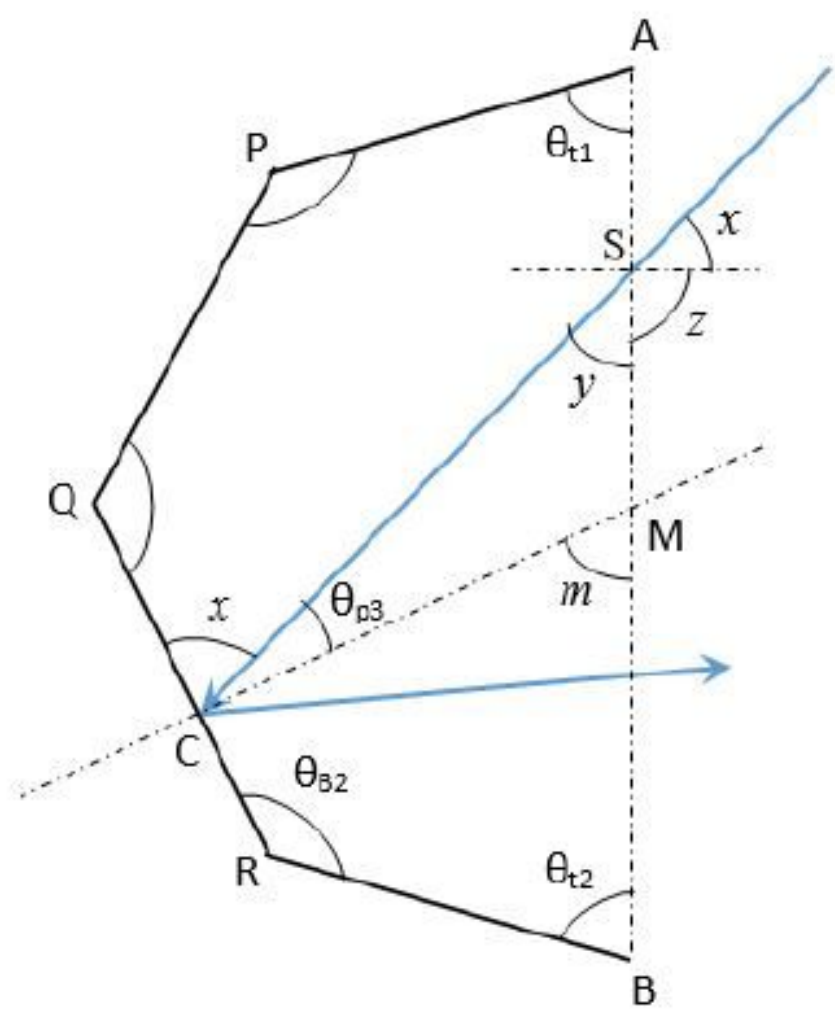

(c)

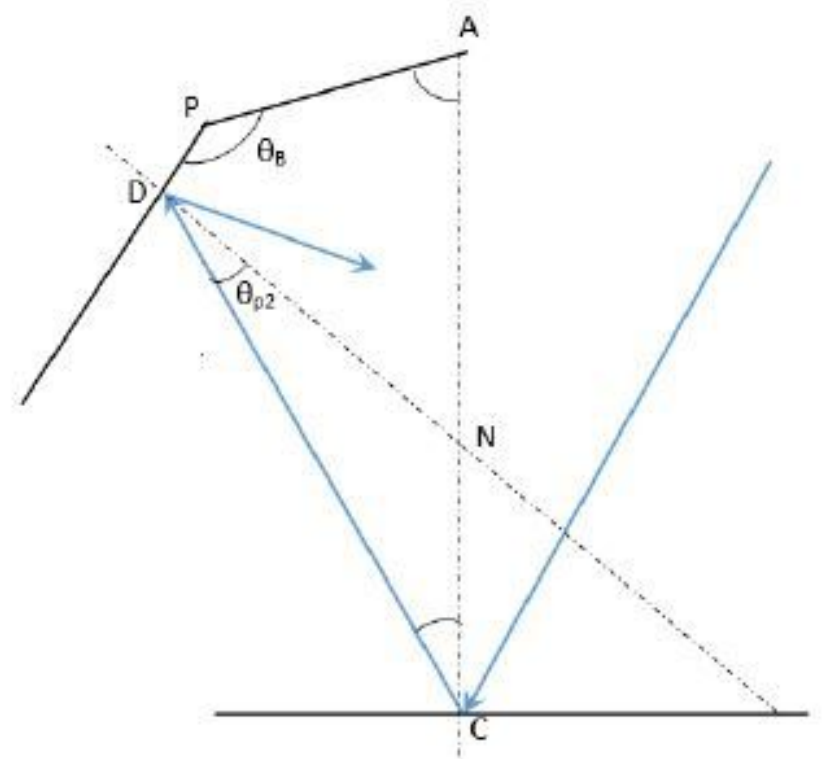

(b)

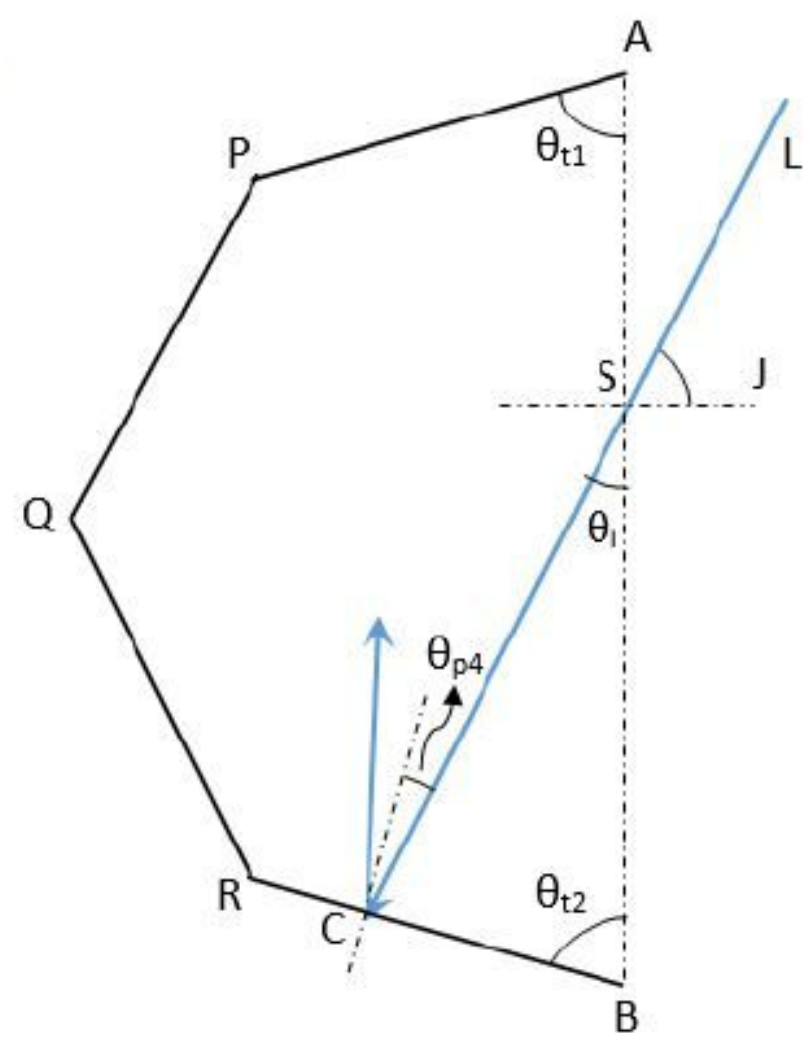

(d)

Figure 2

Schematic representing the light striking angle formation: (a) $\theta p 1$ at first upper interface, (b) $\theta p 2$ at second upper interface, (c) $\theta p 3$ at first lower interface, and (d) $\theta p 4$ at second lower interface w.r.t incoming light incident angle $(\theta i)$ 


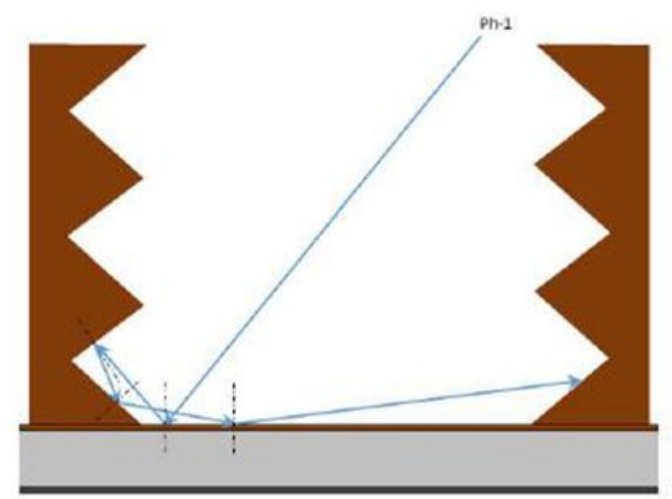

(a)

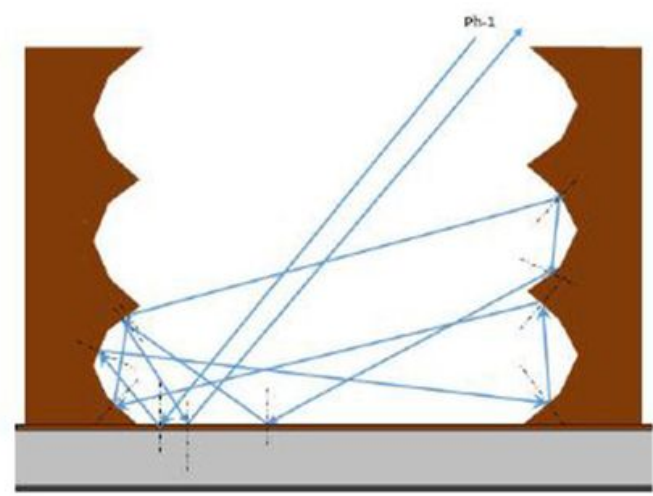

(c)

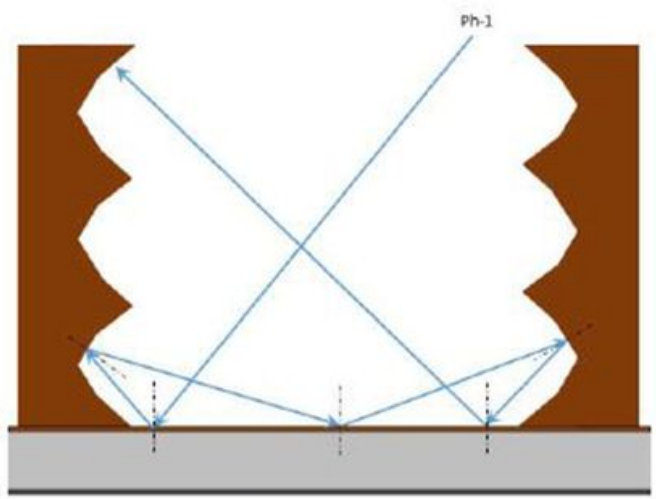

(b)

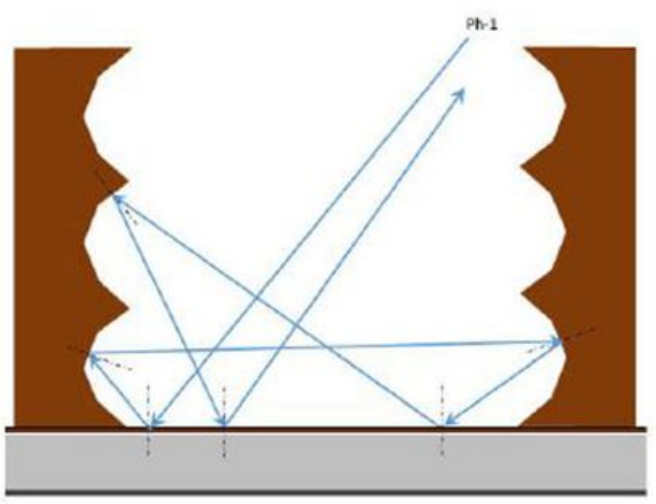

(d)

\section{Figure 3}

Light reflectance pattern within the proposed octagonal cut based nanopillar array structure with centre bending angle ( $\theta B$ ) of: (a) 450, (b) 1000, (c) 1400, and (d) 1600 


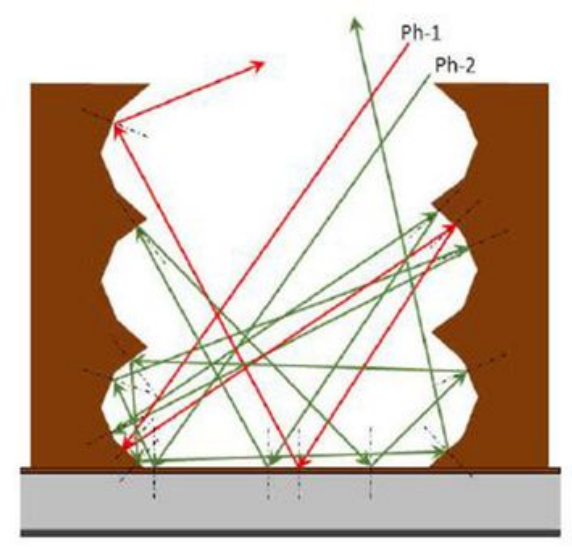

(a)

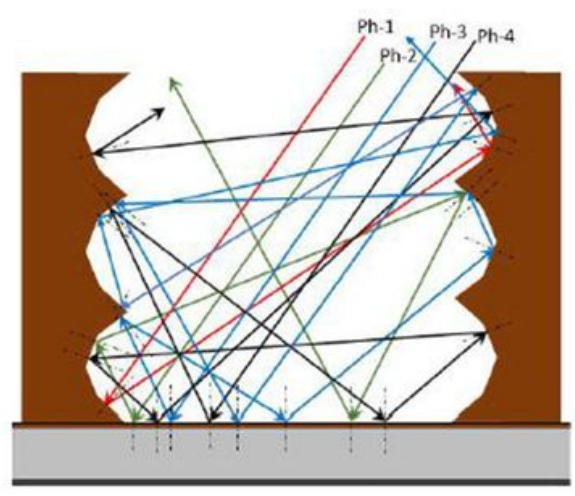

(c)

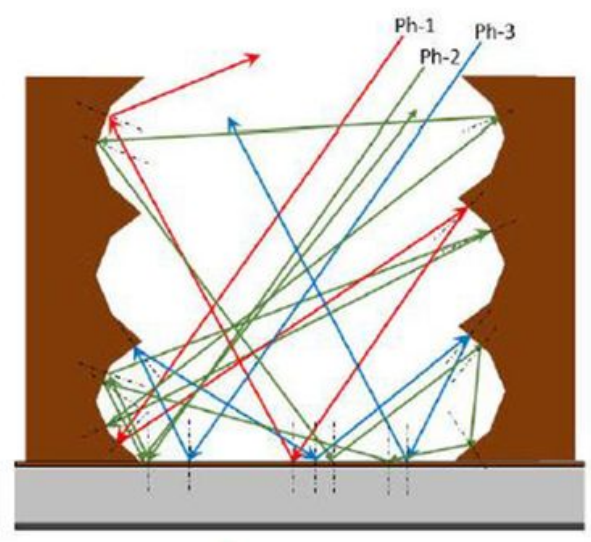

(b)

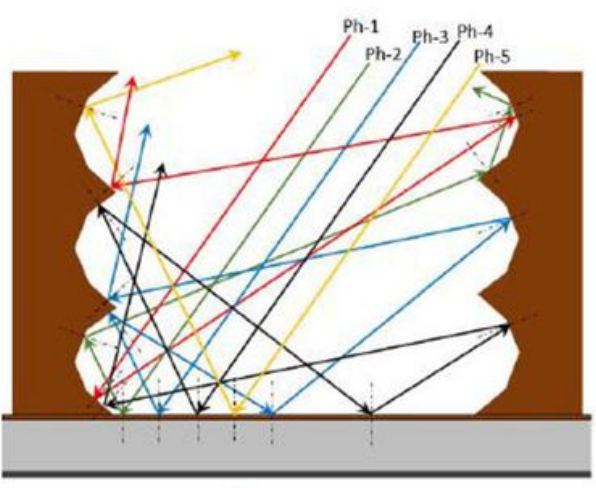

(d)

\section{Figure 4}

Light reflectance pattern within the proposed octagonal cut based nanopillar array structure with varying interpillar spacing: (a) $d<h,(b) h=d,(c) d=[2(h-I \cos (\theta t 1)) / \tan (900-\theta i)] \mid \sin (\theta t 1)$, and (d) $d>$ $[2(\mathrm{hl} \cos (\theta \mathrm{t} 1)) / \tan (900-\theta \mathrm{i})]-\sin (\theta \mathrm{t} 1)$ 


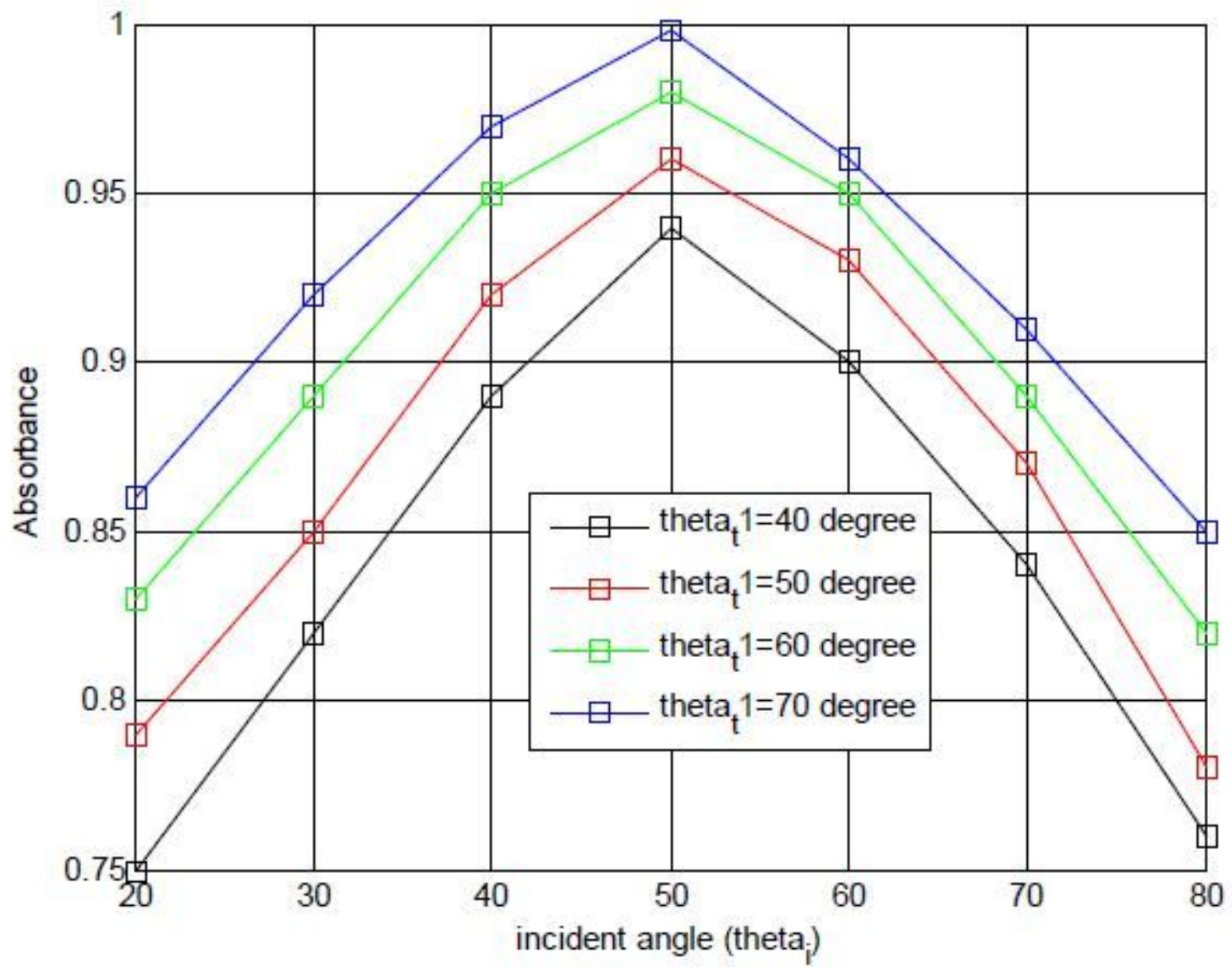

Figure 5

Variation of light absorption with light incident angle at fixed upper tilted angle ( $\theta$ t1) from 400 to 700 


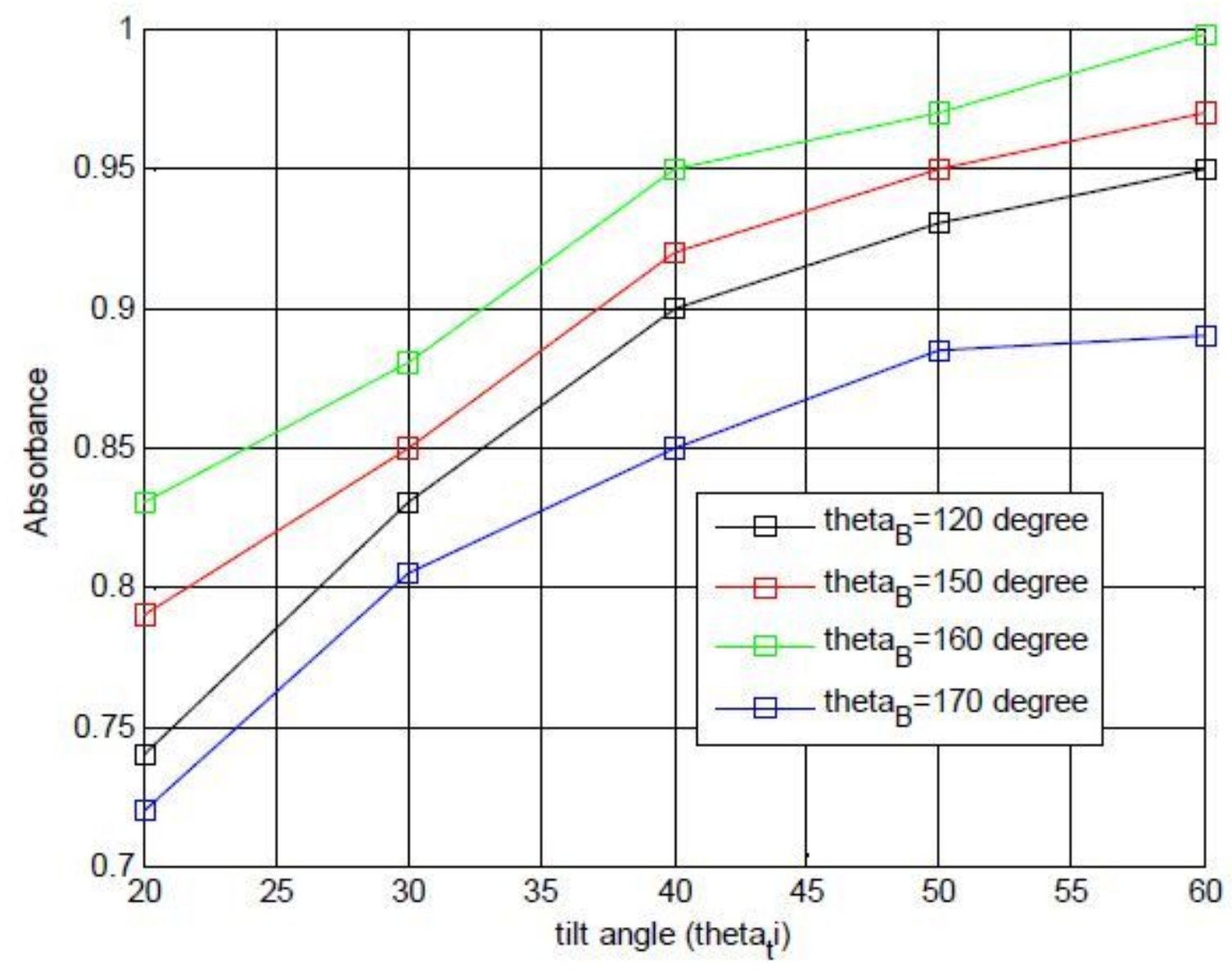

Figure 6

Variation of light absorption with nanopillar upper octagonal cutting angle $(\theta t 1)$ at centre bending angles $(\theta B)$ from 1200 to 1700 for '400'light incident angle 


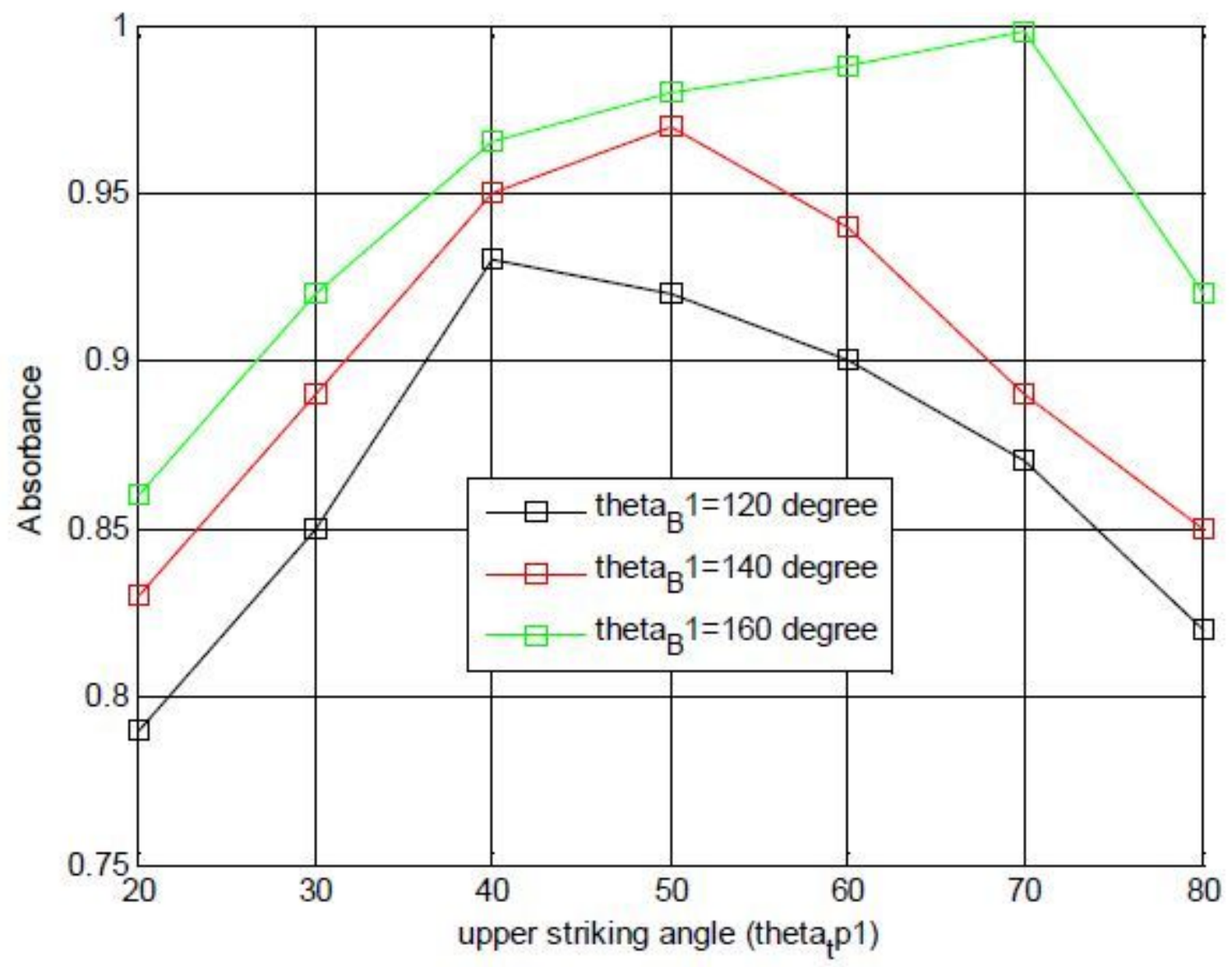

Figure 7

Variation of light absorbance with nanopillar upper cut interface striking angle ( $\theta p 1)$ at fixed upper octagonal bending angles ( $\theta B 1$ ) 1200,1400 and 1600 for '400'light incident angle 


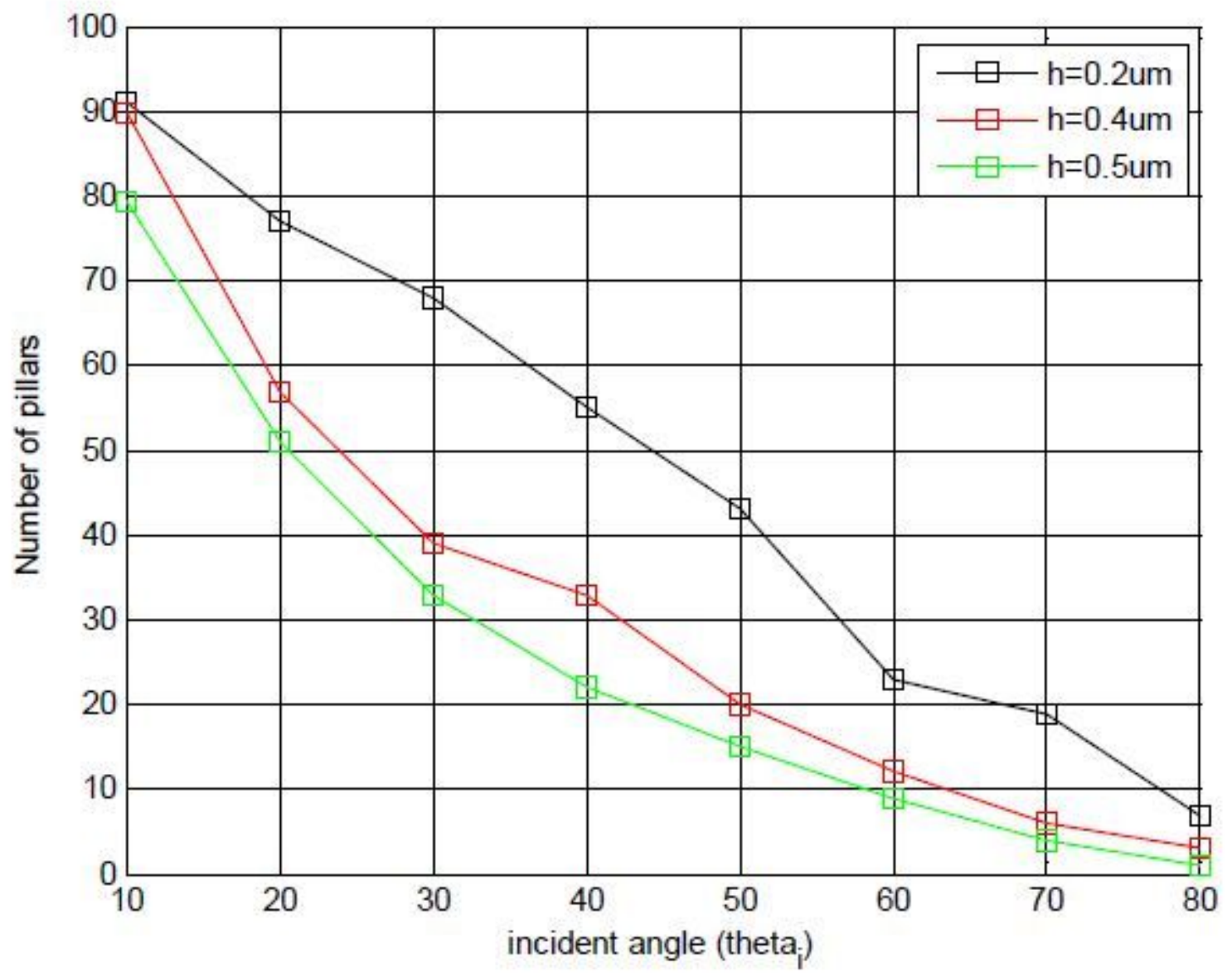

Figure 8

Total number of half octagonal cut based hexagonal nanopillars of varying height from $0.2 \mathrm{um}$ to $0.5 \mathrm{um}$ deployed over '5um x 5um' detector's front surface w.r.t incoming light incident angle ( $\theta \mathrm{i})$ 


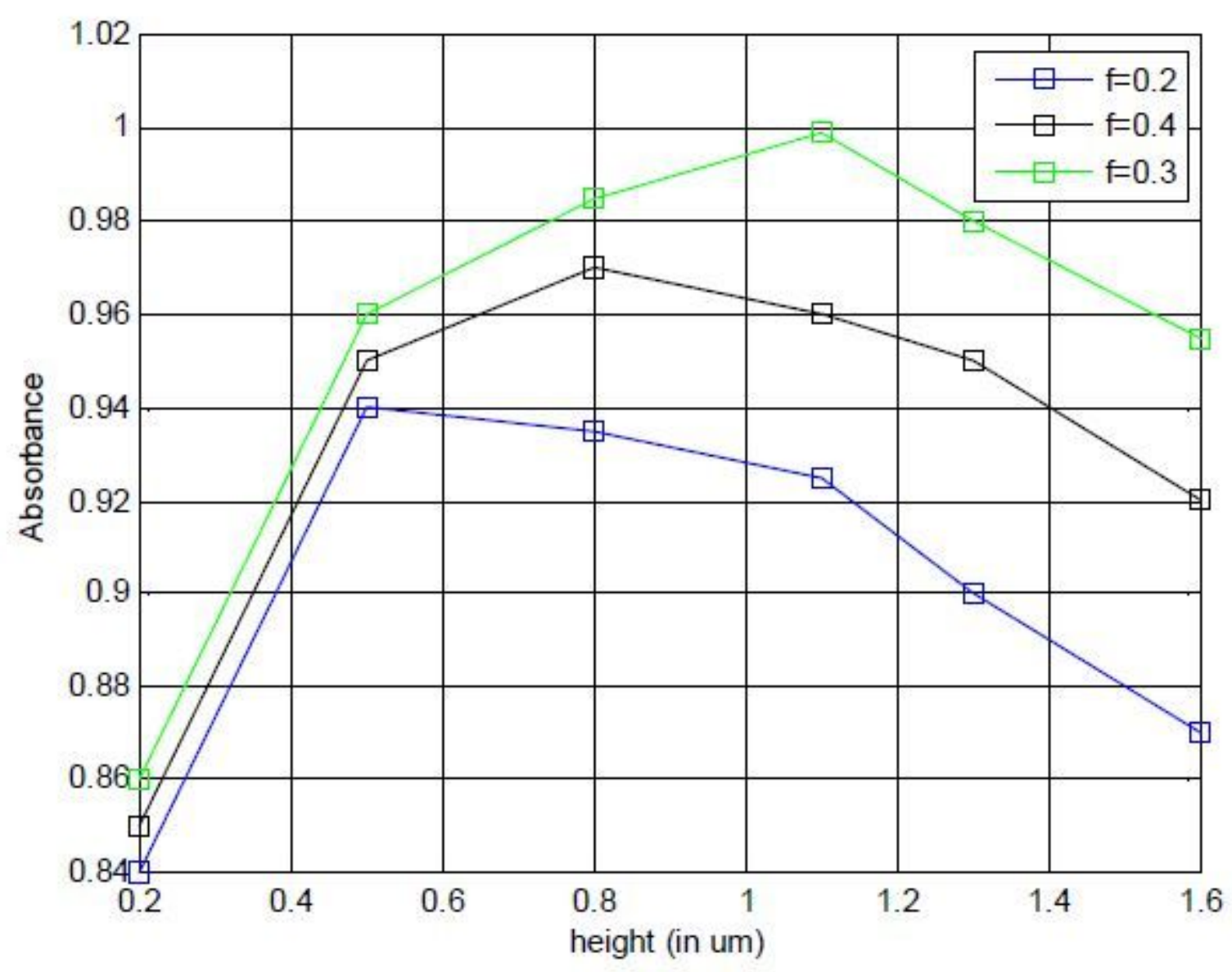

Figure 9

Light absorbance variation obtained within the nanopillar array with increasing pillar height from $0.2 \mathrm{um}$ to 1.6 um at fixed filling ratio from 0.2 to 0.4 


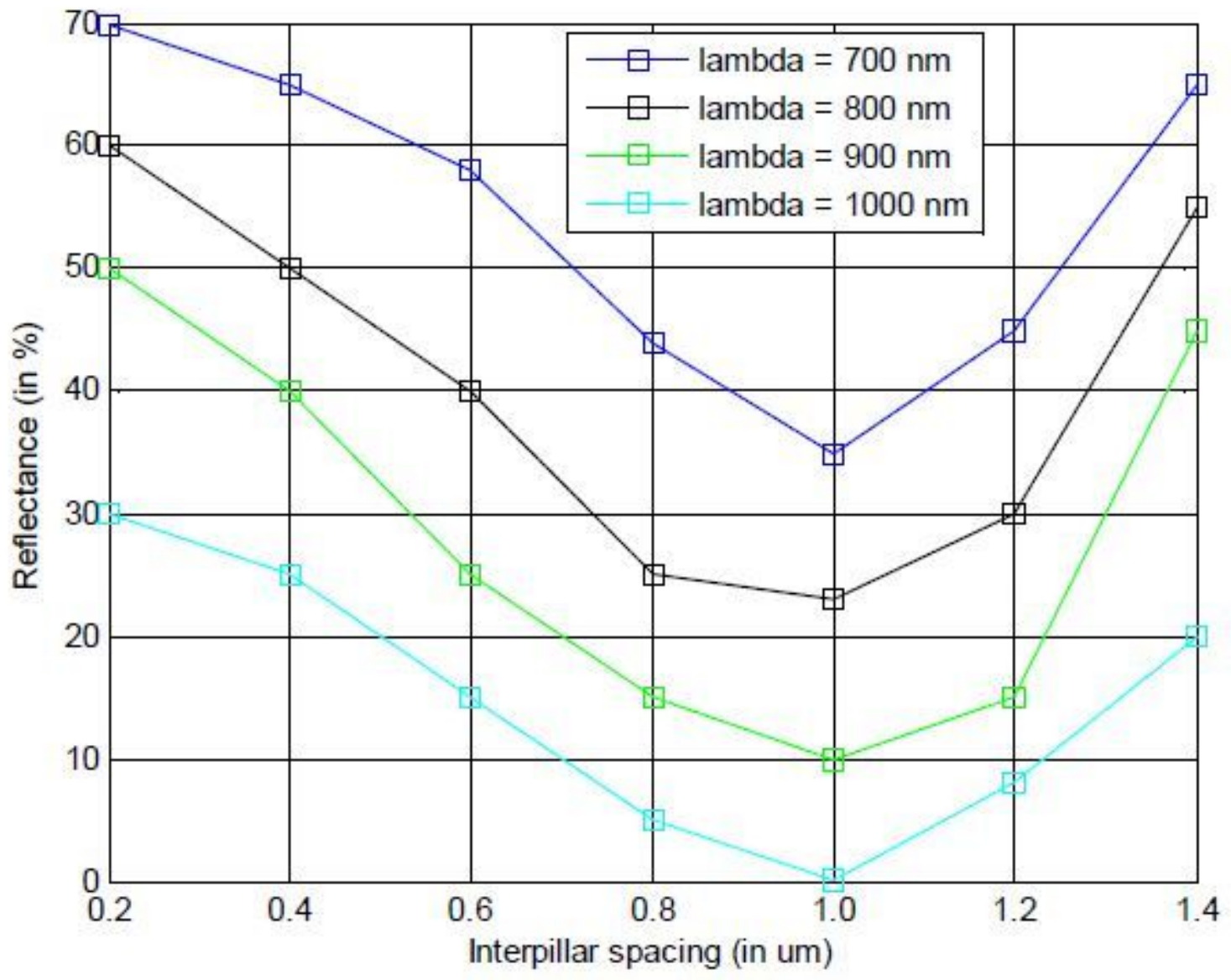

Figure 10

Total light reflectance of 400 incident photons with varying interpillar spacing at different operating wavelength from 0.7 um to 1 um 


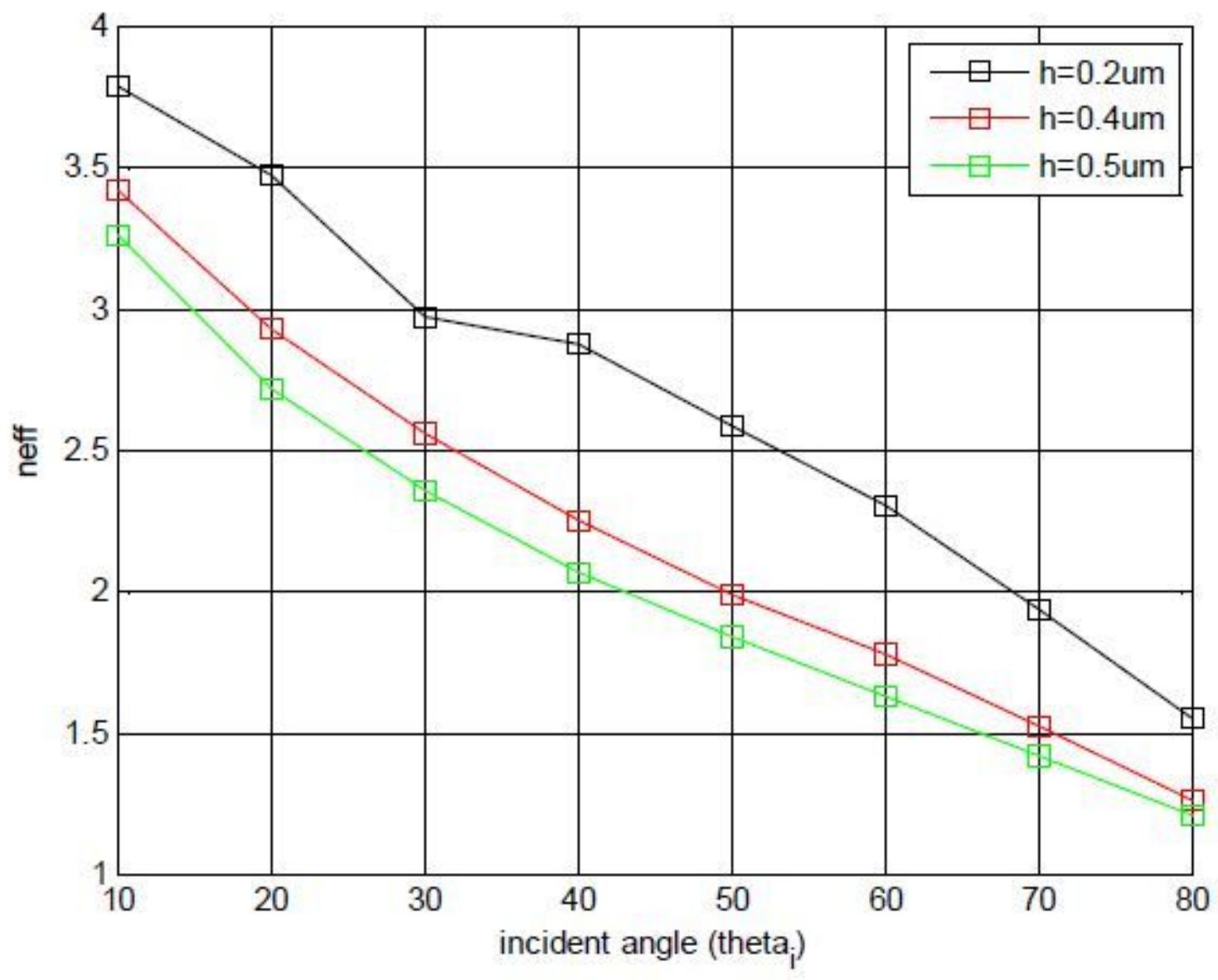

Figure 11

Effective refractive index variation of the proposed nanopillar array according to the light incident angle at fixed nanopillar length from $0.2 \mathrm{um}$ to $0.5 \mathrm{um}$ 


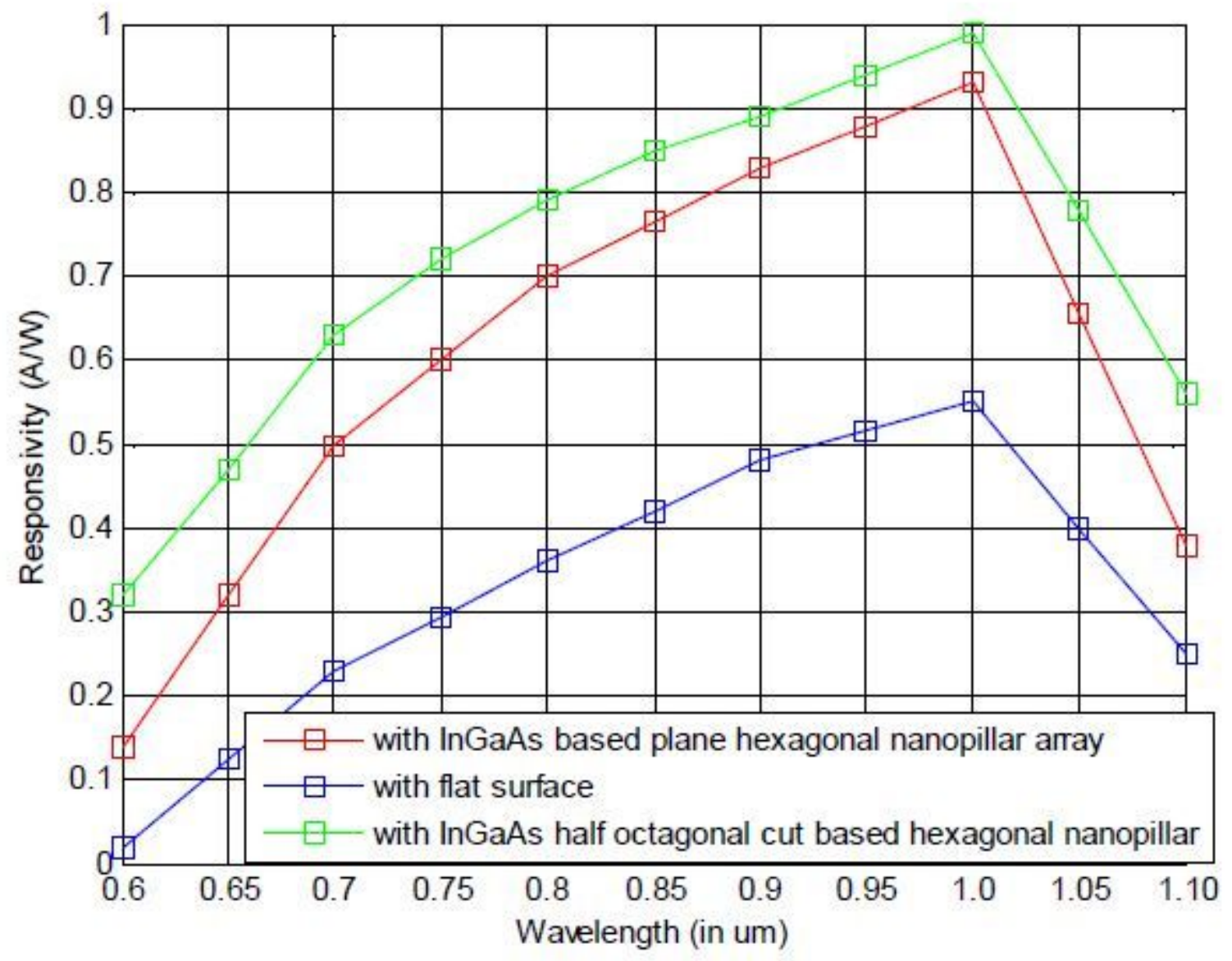

Figure 12

Photoresponse characteristic comparison of the device with proposed half octagonal cut based textured hexagonal nanopillar array surface, planar hexagonal based nanopillar array and flat GaAs surface for various wavelength range with parameters: $h=0.4 u m, f=0.3$ and depletion width $(w d)=0.5 u m$ 


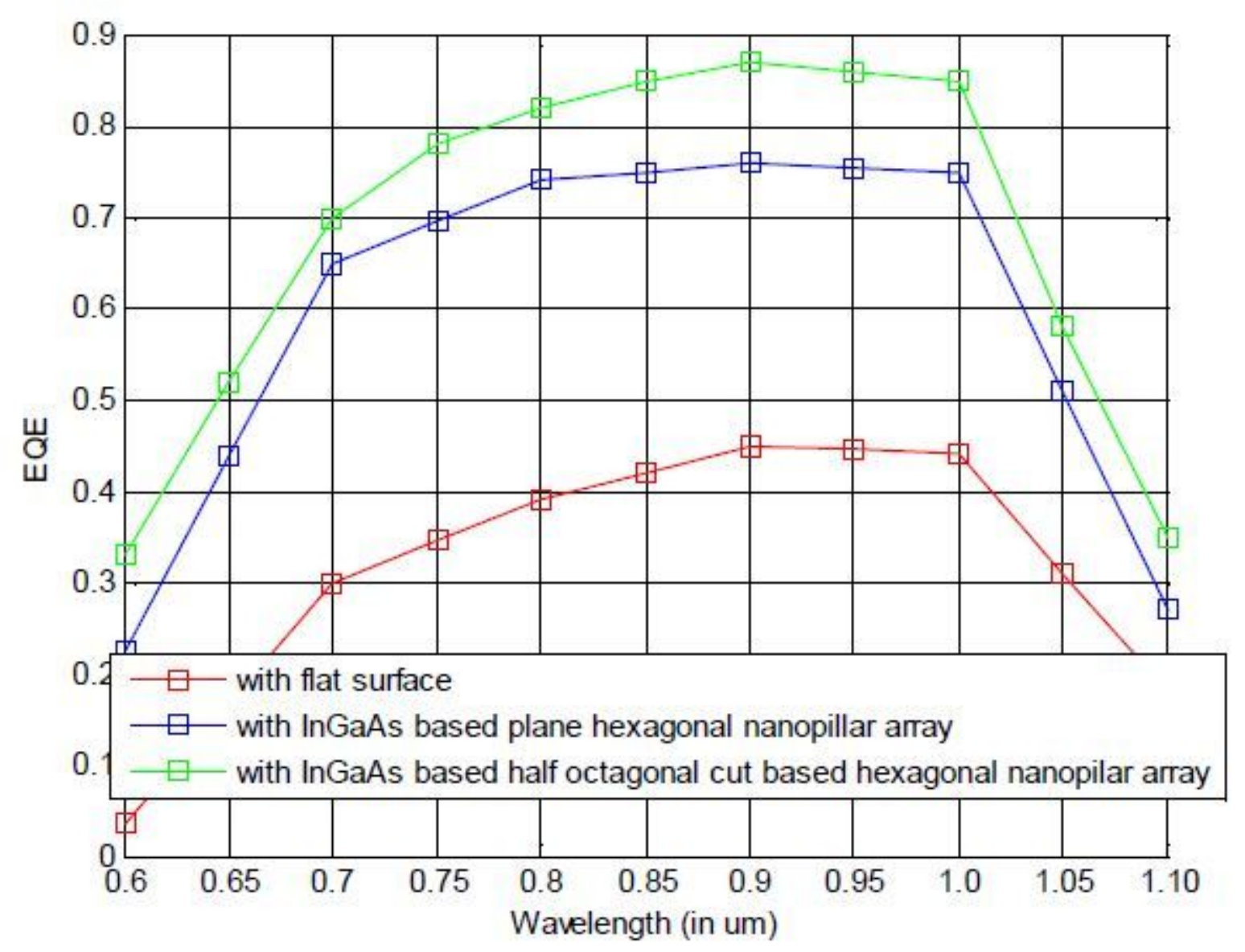

Figure 13

$\mathrm{EQE}(\mathrm{\eta})$ of the photodetector obtained with proposed InGaAs based nanopillar array structure $(\mathrm{h}=0.4 \mathrm{um}, \mathrm{f}=0.3 \mathrm{um}, \mathrm{d}=0.1 \mathrm{um})$, planar $\operatorname{InGaAs}$ based anopillar array structure and flat surface (ws $=0.5 \mathrm{um}$ ) for a wavelength range of $0.6 \mathrm{um}$ to $1.1 \mathrm{um}$ with a $0.5 \mathrm{um}$ depletion width 